University of Nebraska - Lincoln

DigitalCommons@University of Nebraska - Lincoln

2010

Treatment of star catalog biases in asteroid astrometric observations

Steven R. Chesley

Jet Propulsion Laboratory/Caltech, steve.chesley@jpl.nasa.gov

James Baer

James Cook University, jimbaer1@earthlink.net

David G. Monet

United States Naval Observatory

Follow this and additional works at: https://digitalcommons.unl.edu/usnavyresearch

Chesley, Steven R.; Baer, James; and Monet, David G., "Treatment of star catalog biases in asteroid astrometric observations" (2010). U.S. Navy Research. 30.

https://digitalcommons.unl.edu/usnavyresearch/30

This Article is brought to you for free and open access by the U.S. Department of Defense at DigitalCommons@University of Nebraska - Lincoln. It has been accepted for inclusion in U.S. Navy Research by an authorized administrator of DigitalCommons@University of Nebraska - Lincoln. 


\title{
Treatment of star catalog biases in asteroid astrometric observations
}

\author{
Steven R. Chesley ${ }^{\mathrm{a}}$, James Baer ${ }^{\mathrm{b}, *}$, David G. Monet ${ }^{\mathrm{c}}$ \\ a Jet Propulsion Laboratory/Caltech, 4800 Oak Grove Drive, Pasadena, CA 91109, USA \\ ${ }^{\mathrm{b} J a m e s}$ Cook University, School of Engineering and Physical Sciences, Townsville, QLD 4811, Australia \\ ${ }^{\mathrm{c}}$ United States Naval Observatory, Flagstaff, AZ 86001, USA
}

\section{A R T I C L E I N F O}

\section{Article history:}

Received 26 January 2010

Revised 29 May 2010

Accepted 2 June 2010

Available online 11 June 2010

\section{Keywords:}

Asteroids

Comets

\begin{abstract}
A B S T R A C T
In this paper, we discuss the detection of systematic biases in star positions of the USNO A1.0, A2.0, and B1.0 catalogs, as deduced from the residuals of numbered asteroid observations. We present a technique for the removal of these biases, and validate this technique by illustrating the resulting improvements in numbered asteroid residuals, and by establishing that debiased orbits predict omitted observations more accurately than do orbits derived from non-debiased observations. We also illustrate the benefits of debiasing to high-precision astrometric applications such as asteroid mass determination and collision analysis, including a refined prediction of the impact probability of 99942 Apophis. Specifically, we find the IP of Apophis to be lowered by nearly an order of magnitude to $4.5 \times 10^{-6}$ for the 2036 close approach.
\end{abstract}

(c) 2010 Elsevier Inc. All rights reserved.

\section{Introduction}

While future asteroid surveys, such as PanSTARRS, promise to routinely provide observations with uncertainties on the order of 0.1 arcsec (Jedicke et al., 2004), many high-precision astrometric applications, such as mass determination and Yarkovsky analysis, study interactions fixed in the past, or rely upon long observational baselines to model slowly evolving phenomena. So while new observations may be helpful, we must also use contemporaneous and historical observations. Our long-term goal, therefore, is to create a statistical error model of asteroid observations, providing realistic, observatory-specific estimates of error correlations and uncertainties that will allow us to make the best possible use of the existing body of observations.

The astrometric reduction of such observations relies heavily on accurate star catalogs; background stars in an image are identified, and used as references against which the positions of the head and tail of an asteroid trail may be determined. Ideally, star catalogs should be "dense and deep", containing a great many stars of varying brightness distributed throughout the entire sky, allowing high-precision astrometry regardless of asteroid size or location.

In that context, three all-sky US Naval Observatory star catalogs are among the most useful. The USNO A1.0 catalog, introduced in 1996 , contains $488,006,860$ sources down to $V$ magnitude 20 , with an estimated accuracy of 0.25 arcsec (Monet et al., 1998). The A2.0 catalog, introduced in 1998, is an update of A1.0 that moves the

\footnotetext{
* Corresponding author.

E-mail addresses: steve.chesley@jpl.nasa.gov (S.R. Chesley), jimbaer1@earthlink. net (J. Baer).
}

reference frame from the Guide Star Catalog to the ICRF; it contains $526,280,881$ sources (Monet, 1998). The B1.0 catalog, introduced in 2003, contains $1,042,618,261$ objects down to V magnitude 21 , with an estimated accuracy of 0.20 arcsec (Monet et al., 2003).

Note that other catalogs, such as UCAC2 and 2MASS, have smaller estimated position errors; but these catalogs each have limitations relative to B1.0. The UCAC2 catalog, introduced in 2004, contains $48,330,571$ stars with positions accurate to within 0.07 arcsec at the limiting magnitude of 16 ; but it only covers the sky from declination $-90^{\circ}$ to $+40^{\circ}$ (Zacharias et al., 2004). (Note: The recently introduced UCAC3 catalog (Zacharias et al., 2004) covers the entire sky, thus resolving this limitation.) The all-sky 2MASS catalog, introduced in 2003, contains 470,992,970 objects, with positions accurate to within 0.07 arcsec (Skrutskie et al., 2006); but it is limited to $\mathrm{V}$ magnitude 17 , meaning that reference stars may be overexposed in images of small asteroids.

While creating the first iteration of a statistical error model of asteroid observations, we detected significant biases in the residuals of the numbered asteroids, which were traced to biases in the star catalogs from which those observations were reduced.

After describing our discovery and investigation of these biases (Section 2), we will describe the available asteroid astrometric data (Section 3). Next, we present a technique to debias these observations (Section 4), and demonstrate that it substantially eliminates systematic errors in orbit fits of the numbered asteroids (Section 5). We validate this new debiasing technique by demonstrating that it produces orbits that predict better, which is the crucial test of the quality of an orbit estimate (Section 6). Finally, we illustrate the application of catalog debiasing to issues such as estimating the mass of perturbing asteroids, and assessing the probability of an impact for potentially hazardous asteroids (Section 7). 
With the catalog biases resolved, a future paper will describe the subsequent development of a statistical error model for asteroid observations, demonstrate its validity, and detail its applications.

\section{Evidence for bias}

Star catalog biases were encountered during the development of a statistical error model for astrometric asteroid observations, as described in Carpino et al. (2003). Such a model requires that the observational residuals be unbiased, normally distributed, and uncorrelated.

In testing the first iteration of our error model, however, it became clear that these assumptions were being violated.

As illustrated in Fig. 1, for instance, probability distributions of the nominal declination residuals for the numbered asteroids revealed a clear bias of approximately +0.16 arcsec. Significantly, no such bias is evident in the right ascension residuals; this led us initially to believe that only the declination observations were affected.

Additionally, we calculated a kurtosis of approximately 4.6 for both the RA and the DEC residual probability distributions depicted in Fig. 1; this compares to a kurtosis of 3.0 for the standard normal distribution, indicating that the RA and DEC distributions are slightly peaked, thus deviating somewhat from the expected normal distribution. Adjusting the chi-square observation rejection threshold in the orbit determination algorithm failed to reduce either kurtosis to expected levels. (Note: The definition of kurtosis used in this paper results in a normal distribution having a kurtosis of 3.0.)

Finally, as will be discussed in Section 5.3, the residuals from closely-spaced observations of the same asteroid made by the same observatory appeared highly-correlated. While the correlations associated with each specific observatory differed somewhat in magnitude, they remained significant even for observations separated by several days.

Based on these data, we hypothesized that there were systematic declination biases in the star catalogs used to reduce the asteroid observations. And as we investigated further, we encountered evidence outside of our own work.

Fig. 2, dating to mid-2008, depicts histograms of the postfit means of right ascension and declination residuals for the 1649 numbered asteroids under automated orbit maintenance at that time. While the right ascension histogram shows a symmetric distribution with a mean of 0.004 arcsec and a standard deviation of

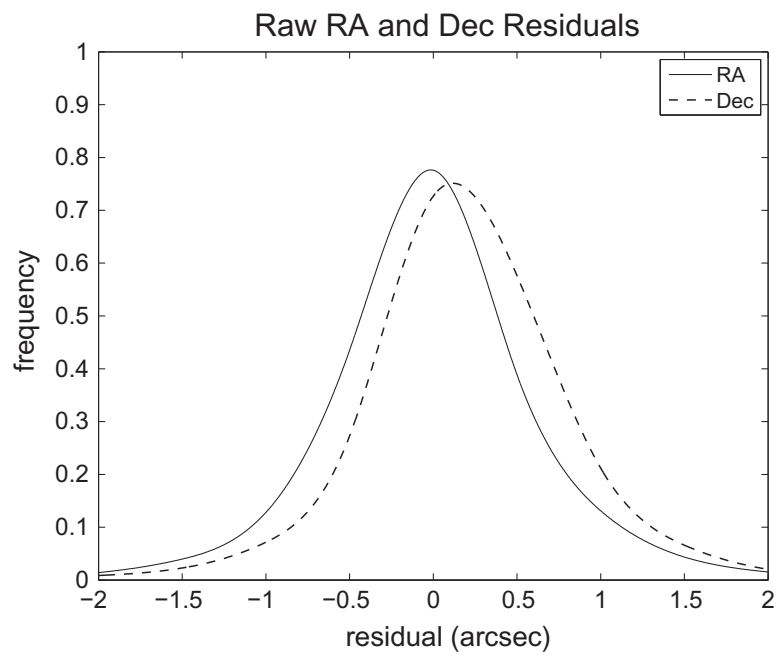

Fig. 1. Nominal residuals of the numbered asteroids, illustrating DEC bias.
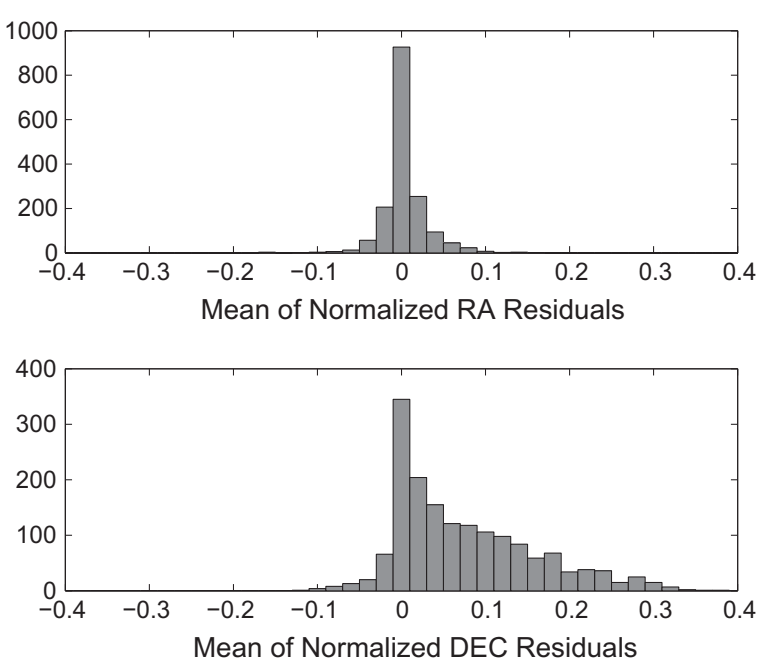

Fig. 2. Mean of normalized postfit residuals for 1649 numbered asteroids under automated orbit maintenance as of mid-2008, including all numbered NEAs, plus a handful of other targets of interest, such as space mission targets. The residuals were normalized by dividing each residual by its corresponding weight; and since the vast majority of observations are weighted at 1 arcsec, the abscissa is approximately in arcsec.

0.057 arcsec, the declination histogram is decidedly nonGaussian and asymmetric, with a mean of 0.074 arcsec and a standard deviation of 0.095 arcsec.

Upon analysis of the Rosetta spacecraft's encounter with Asteroid 2867 Steins, Morley (T. Morley, private communication) independently deduced a bias of +0.212 arcsec in the declination observations of that asteroid. However, to fully account for the observed encounter geometry, a right ascension bias of +0.092 arcsec was also necessary. This was the first indication that a bias was present in both coordinates. As Morley noted, a systematic bias in the right ascension observations of asteroids would not be immediately obvious, since a least-squares orbit determination algorithm would simply rotate the calculated orbit in the equatorial frame about the celestial pole to minimize the RMS error, thus eliminating the evidence of a net right ascension bias. Since none of the other orbital parameters could be manipulated so as to eliminate the declination bias, it appeared to be the only systematic error in the observations.

As will be described more fully in Section 7.2.1, Mauna Kea Observatory (MPC observatory code 568) independently noted a persistent positive declination bias in observations of 99942 Apophis (D. Tholen, private communication). In the discussions that followed, Tholen referred us to da Silva Neto et al. (2005), who had observed a mean declination bias of approximately 0.11 arcsec in the USNO B1.0 positions of the optical counterparts of 64 ICRF sources. Using the more accurate UCAC2 catalog as a reference, comparisons of the positions of stars appearing in both B1.0 and UCAC2 were used to derive local corrections to the B1.0 positions; implementing these local corrections, the B1.0 declination biases for the ICRF sources were reduced to approximately 0.03 arcsec. In time, this idea proved most useful.

\section{The asteroid astrometric data}

The Minor Planet Center (MPC), hosted by the Smithsonian Astrophysical Observatory at Harvard Univ., Cambridge, Mass., operates under the auspices of Division III of the International Astronomical Union as the clearinghouse for asteroid astrometric measurements, both optical and radar (Williams, 2009). As a part of this function, the MPC archives and distributes the available data on intervals of approximately one month. 
Table 1

Optical observation data types.

\begin{tabular}{llr}
\hline Type & MPC flag & \multicolumn{1}{l}{ Count } \\
\hline CCD & C, c & $58,083,989$ \\
Former B1950.0 & A & 662,222 \\
Photographic & & 303,325 \\
Transit circle & T & 26,968 \\
Micrometer & M & 12,081 \\
Approximate & X & 10,763 \\
Hipparcos & H & 5494 \\
Occultation & E & 991 \\
Satellite & S & 277 \\
Roving observer & $\mathrm{V}$ & 177 \\
Mis-tagged obs. & $\mathrm{b}$ & 1 \\
Total & - & $59,106,288$ \\
\hline
\end{tabular}

We have based the present study on the complete MPC astrometric data set dated 2008 December 12. In this data set there are 59,106,288 optical observations of asteroids, a number that includes 48,807,317 optical observations of 202,855 numbered asteroids and an additional 10,298,971 optical observations of asteroids temporarily designated but not yet numbered. There are also radar delay and Doppler observations, which we neglect for the present discussion. The optical data set is dominated by CCD observations, which comprise $98.3 \%$ of the observations.

The MPC astrometric data are stored in 80-column ASCII records. A nearly complete description of the data format is available on the MPC website, ${ }^{1}$ which we briefly summarize here. There is one record per observation, except for Satellite and Roving observations, which require a second observation record to describe the position of the observer. The primary information on the optical astrometry data record is the observation time and sky position, given as (J2000) right ascension and declination. The measurement technique, or observation type, is recorded by a code in column 15 , the counts for which are given in Table 1 . The observatory code in columns $78-80$ is a three-character identifier that uniquely identifies the observing location, and sometimes the observing program. (In cases where multiple observing programs share the same observatory code, e.g., Mauna Kea or Mt. Palomar, there may be an additional field that records the observing program.) The observatory codes and the names and locations of the associated observatories are tabulated by the MPC. ${ }^{2}$ In this paper, for brevity, we will often refer to the observatories only by their observatory code. There is also a field for the observation's Minor Planet Circular or Supplement reference, and a field that allows the observer to provide a terse annotation regarding the observation quality or reduction technique. Photometry information may also be part of the record.

For the present effort, the most important part of the observation record is the undocumented annotation, in column 72 , of the star catalog that was used in the reduction of the astrometry (Gareth Williams, private communication). Table 2 lists the field values and their meanings, along with the number of CCD observations for each field value. An inspection of the data indicates that the MPC did not start recording the catalog in the observation record until mid-2001, and even after that many observers did not report the catalog information to the MPC. Thus for about $17 \%$ of the astrometry there is no catalog information available on the MPC data record. Fortunately, much of the missing catalog information is from only a few observatory codes, and for data from prior to mid-2001. Thus, although the MPC did not record the information, some of the observing programs still have their observation logs from that period and so we were able to supply catalog

\footnotetext{
${ }^{1}$ http://www.cfa.harvard.edu/iau/info/ObsFormat.html.

2 http://www.cfa.harvard.edu/iau/lists/ObsCodesF.html.
}

Table 2

Catalog flags found for CCD observations on MPC data file.

\begin{tabular}{|c|c|c|}
\hline Catalog & MPC flag & Count \\
\hline USNO A1.0 & $\mathrm{a}$ & 173,556 \\
\hline USNO SA1.0 & $\mathrm{b}$ & 31,044 \\
\hline USNO A2.0 & c & $26,071,594$ \\
\hline USNO SA2.0 & d & $1,327,783$ \\
\hline UCAC1 & $\mathrm{e}$ & 243,111 \\
\hline Tycho-1 & $\mathrm{f}$ & 0 \\
\hline Tycho-2 & $\mathrm{g}$ & 299,648 \\
\hline GSC 1.0 & $\mathrm{~h}$ & 0 \\
\hline GSC 1.1 & $\mathrm{i}$ & 10,055 \\
\hline GSC 1.2 & $\mathrm{j}$ & 11,665 \\
\hline GSC 2.2 & $\mathrm{k}$ & 180 \\
\hline $\mathrm{ACT}$ & 1 & 13,866 \\
\hline GSC ACT & $\mathrm{m}$ & 364,575 \\
\hline TRC & $\mathrm{n}$ & 0 \\
\hline USNO B1.0 & 0 & $6,799,942$ \\
\hline PPM & $\mathrm{p}$ & 0 \\
\hline UCAC2-beta & $q$ & 0 \\
\hline UCAC2 & $\mathrm{r}$ & $12,315,889$ \\
\hline USNO B2.0 & s & 445 \\
\hline UCAC3-beta & $\mathrm{t}$ & 0 \\
\hline UCAC3 & $\mathrm{u}$ & 0 \\
\hline NOMAD & $\mathrm{v}$ & 2763 \\
\hline CMC & w & 337,053 \\
\hline Hip 2 & $\mathrm{x}$ & 0 \\
\hline GSC (generic) & $\mathrm{z}$ & 1537 \\
\hline Unspecified & - & $10,079,283$ \\
\hline Total & - & $58,083,989$ \\
\hline
\end{tabular}

Table 3

Observer-supplied catalog information.

\begin{tabular}{lll}
\hline Obs. code & Time frame & Catalog \\
\hline 704 & Before 2000-January-01 & USNO A1.0 \\
704 & After 2000-January-01 & USNO A2.0 \\
691 & 1991-August-31-1999-September-28 & GSC 1 \\
691 & 1999-September-29-2000-December-21 & USNO A1.0 \\
691 & 2000-December-22-2006-December-26 & USNO A2.0 \\
703 & Before 2005-January-01 & USNO A2.0 \\
\hline
\end{tabular}

info for $59 \%$ of the untagged astrometry. Table 3 lists the rules that we have applied to otherwise untagged astrometry.

Many of the individual catalog tags given in Table 2 can be grouped together for our purposes. For instance, the USNO SA2.0 catalog is merely a subset of USNO A2.0, and UCAC2 is an extension to UCAC1. We have merged the various column-72 symbols as detailed by Table 4 . We note that over $90 \%$ of the astrometry is comprised from four catalogs, USNO A1.0, USNO A2.0, USNO B1.0, and UCAC. An additional 7.2\% still have catalog unknown, although most of these are from major NEO survey programs, and so these also are probably dominated by the same four catalogs. As we discuss below, one can infer which catalog was used in such cases by a statistical analysis of the residuals. Fig. 3 depicts the history of catalog use for the data given in Table 4. Some catalogs appear earlier than their release date because older images were in some cases remeasured with new software and star catalogs. USNO A2.0 has been used heavily, with more than 2 million observations every year since 2000, which happened to be the last year that USNO A1.0 saw extensive usage. But starting in 2005, the UCAC and USNO B1.0 catalogs have together accounted for the majority of reported astrometry.

\section{Two approaches to debiasing}

There are two obvious way to quantify the effect of star catalog bias in asteroid orbit determination. One can proceed indirectly, 
Table 4

Merged catalog counts.

\begin{tabular}{lllr}
\hline Catalog & MPC flags & Count & $\%$ \\
\hline USNO A2.0 & c, d & $30,786,427$ & 53.0 \\
UCAC & e, q, r, t, u & $12,559,000$ & 21.6 \\
USNO B1.0 & o, s & $6,800,387$ & 11.7 \\
Unknown & - & $4,171,601$ & 7.2 \\
USNO A1.0 & a, b & $2,205,452$ & 3.8 \\
GSC 1 & h, i, j, z & 543,037 & 0.9 \\
GSC ACT & m & 364,575 & 0.6 \\
CMC & w & 337,053 & 0.6 \\
Tycho & f, g & 299,648 & 0.5 \\
ACT & l & 13,866 & 0.0 \\
NOMAD & $\mathrm{v}$ & 2,763 & 0.0 \\
GSC 2 & $\mathrm{k}$ & 180 & 0.0 \\
Total & - & $58,083,989$ & - \\
\hline
\end{tabular}

through a systematic study of the postfit residuals of astrometric asteroid observations, or directly, by comparing star catalog positions with those in a more accurate and precise reference. Since the problem first arose for us from the perspective of asteroid residuals, we began with the first approach.

\subsection{Debiasing from indirect reference to postfit astrometric residuals}

As shown in Fig. 1, the observations of numbered asteroids demonstrated a clear observational bias in declination; but no such bias appeared in right ascension. We therefore began by debiasing only the declination coordinates. After calculating the orbits of the numbered asteroids and compiling the "observed-predicted" residuals, we divided the sky into 18 zones of declination, each $10^{\circ}$ wide; within each zone, we calculated the mean declination biases for USNO A1.0, A2.0, B1.0, UCAC2, and observations of various eras whose reduction catalogs were unknown. We then created best-fit polynomials for each of these groups to model the declination biases in each zone, and used them to debias the observations for the next iteration.

Initially, the approach seemed promising; the biases resulting from the second iteration were reduced to one third of their previous values. However, in order to achieve these reductions, we had to apply significant corrections (on the order of 0.35 arcsec) to the

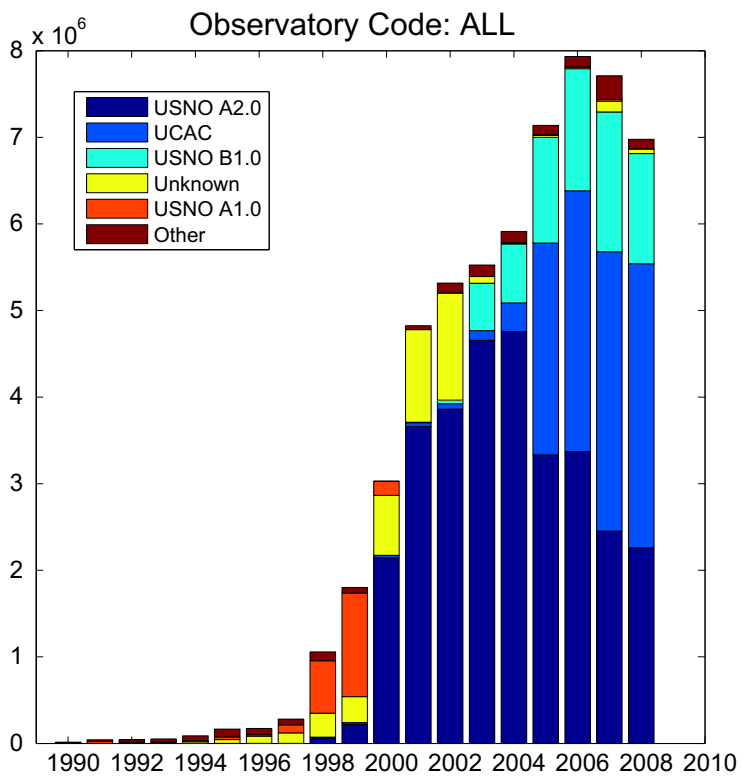

Fig. 3. Year-by-year counts of star catalog usage for CCD astrometry.
UCAC2 declination observations. Given the stated accuracy of the UCAC2 star positions, this was clearly unrealistic. We concluded that the biases in the other catalogs were likely resulting in bestfit orbits that made the UCAC2 residuals appear much larger than their intrinsic errors. Therefore, we decided to repeat the second iteration, but with no corrections applied to the UCAC2 derived positions.

The results from the revised second iteration showed significant improvement; for most of the observation sets, the biases had been reduced to below 0.06 arcsec. And yet the biases in the UCAC2 derived observations remained above 0.2 arcsec. A third iteration yielded little further reduction.

By this point, additional analysis had demonstrated that the asteroid residuals did indeed contain a right ascension bias; and we suspected that our failure to account for this was responsible for the slow convergence in eliminating the observed bias in the UCAC2 residuals. We therefore implemented a two-dimensional bias model for all future iterations, with a grid spacing of $1 \mathrm{~h}$ in right ascension and $10^{\circ}$ in declination. As before, the mean observed bias in each grid square was used to debias the observations in the next iteration. But unfortunately, two further iterations yielded little further improvement; the biases in the UCAC2 derived observations remained near 0.2 arcsec.

Despite these difficulties, the bias corrections that we derived through this indirect approach do share many of the gross properties seen in the corresponding (and much more detailed) sky maps derived from the direct approach seen in the next section. In retrospect, we believe that, had we started with a two-dimensional bias model in the first place, perhaps with smaller bins on the sky, this iterative technique might have yielded adequate results; but we do not believe that it can be as effective as the direct approach that we have developed, which we describe now.

\subsection{Debiasing from direct reference to star catalogs}

An assessment of the systematic errors in star catalog astrometry is the key ingredient in the direct approach to quantification of systematic errors in asteroid astrometry from this source. Thus we needed to produce a spatially resolved astrometric comparison of various catalogs that have been used as reference catalogs for the astrometry of solar system objects. Because of its wide dynamic range and its rigorous ties to the ICRS (Arias et al., 1995) using the Tycho-2 catalog (Høg et al., 2000), the 2MASS star catalog (Skrutskie et al., 2006) was chosen as the catalog to which all others were compared. According to its documentation, the 2MASS systematic position errors are $70-80$ milliarcsec with respect to the ICRS. The 2MASS catalog does not contain proper motions, but the mean epoch of the 2MASS observations is very close to 2000.0. Hence, the comparison of all of the catalogs ignored proper motions.

To achieve the desired spatial resolution, the JPL HEALPix ${ }^{3}$ tessellation (Górski et al., 2005) was used. Adopting SIDE $=64$ with the HEALPix package produces 49,152 tiles on the sky, called HEALPix, each with an area just a bit smaller than one square degree. This seems to be a reasonable compromise between high resolution and having enough stars in each tile. Given this tessellation scheme, the tile number for each entry of each star catalog was computed, and the catalogs were sorted on this index to expedite the calculation.

The numerical processing was the same for all catalogs under consideration. For each HEALPix, the stars in the catalog and in 2MASS were extracted, and a spatial correlation using a radius of 2.0 arcsec was used to identify stars in common. The size of the radius was chosen to be large enough to accommodate the expected

\footnotetext{
${ }^{3}$ http://healpix.jpl.nasa.gov.
} 
systematic differences (up to 1.0 arcsec), but small enough to minimize the number of spurious correlations. Once the pairs were identified, the mean and standard deviation in the right ascension and declination directions were computed. These provide a measure of the systematic and random local position errors. Note that the calculation was done in the tangent plane. For each HEALPix, a tangent point at the center of the tile was adopted, and the tangent plane positions for the catalog and 2MASS stars were computed. Hence, the resulting astrometric shifts and dispersion should mimic those needed to correct the analysis of observational data.

We performed the analysis for five of the catalogs listed in Table 4: USNO A1.0, USNO A2.0, USNO B1.0, UCAC2, and Tycho. The output of the comparison of these catalogs with 2MASS was a single file containing 49,152 lines each with the nominal position of the HEALPix on the sky and values for the mean and standard deviation for the astrometric offset (excepting for UCAC2 which does not cover the entire sky). While the primary use of the comparison file is intended to be a scheme by which individual observations can be corrected to the astrometric reference frame defined by the 2MASS catalog, various visualization tools were used to understand the gross properties of the various catalogs.

Fig. 4 includes plots of the probability density of the mean differences between the various catalogs and 2MASS among the 49,152 HEALPix cells. Table 5 lists the mean and standard deviation of the same distributions. From these, we observe that the precision and accuracy of the Tycho and UCAC catalogs are comparable, and vastly superior to the other catalogs. Indeed, the differences with respect to 2MASS appear small enough that it is difficult to know whether they should be attributed to the subject catalogs, to 2MASS or whether the differences are a blend of errors from both sides of the comparison.

But, Tycho and UCAC have relatively few stars and are too sparse for most asteroid astrometry work, and so much of the asteroid astrometry has been and continues to be measured against the much more densely populated USNO family of catalogs. Here we see that the USNO A1.0 has relatively large dispersions, of order 400 mas, but overall it exhibits a relatively modest bias. On the other hand, the USNO A2.0 and B1.0 catalogs reveal significant biases overall, especially in declination, with mean offsets of 142 mas and 126 mas, respectively. These values are comparable to the standard deviations of the samples. In right ascension, B1.0 appears relatively unbiased overall, while A2.0 does show a modest net RA bias.
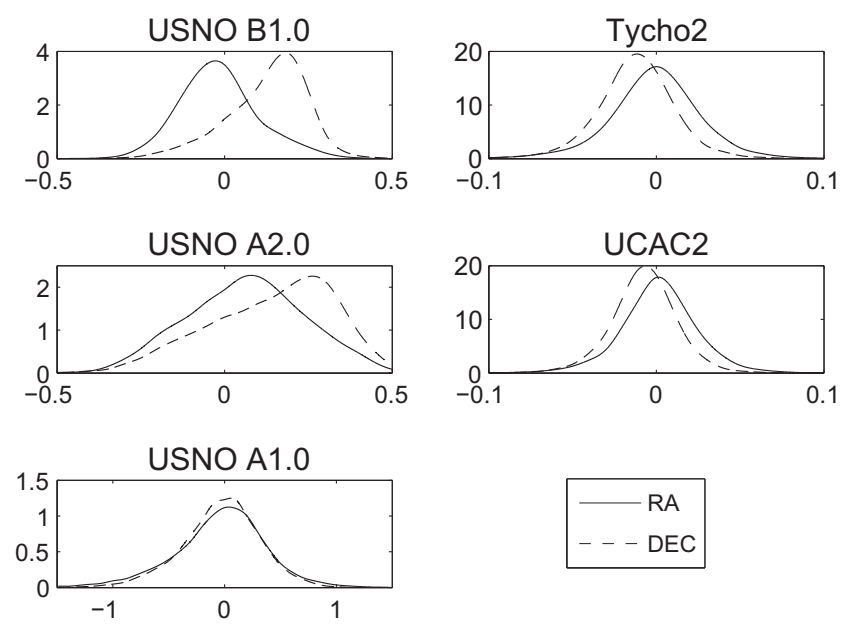

Fig. 4. Probability densities of inter-catalog systematic errors, as compared to the 2MASS catalog. For each plot, the abscissa is the difference in arcsec between the given catalog and 2MASS and the ordinate is the associated probability density in $\operatorname{arcsec}^{-1}$. Note that the plots are not all on the same scale.
Table 5

Inter-catalog systematic errors, with respect to 2MASS.

\begin{tabular}{lcc}
\hline \multirow{2}{*}{ Catalog } & \multicolumn{2}{l}{ Mean \pm std. dev. } \\
\cline { 2 - 3 } & RA (mas) & DEC (mas) \\
\hline Tycho-2 & $-1 \pm 28$ & $-12 \pm 24$ \\
UCAC2 & $2 \pm 23$ & $-7 \pm 20$ \\
USNO B1.0 & $-16 \pm 123$ & $126 \pm 123$ \\
USNO A2.0 & $63 \pm 180$ & $142 \pm 189$ \\
USNO A1.0 & $-41 \pm 419$ & $-34 \pm 352$ \\
\hline
\end{tabular}

Of course, the discussion so far does not reveal anything about the structure on the sky. If half of the sky has a positive offset and half of the sky has a negative offset then the mean offset could still be small. To address this question, Fig. 5 depicts sky maps of the RA and DEC differences with respect to 2MASS for the five catalogs under consideration; while Fig. 6 depicts the standard deviations in those RA differences. Note first that the color scale is different for different catalogs in order to show as much of the structure in the catalog bias as possible. In these plots, regions with relatively small biases are colored green, while red regions show positive bias and blue regions have negative bias. The USNO A1.0 catalog does show significant structure, despite its rather innocuous presentation in Fig. 4, and yet the hemispheric differences are relatively modest. On the other hand, the USNO A2.0 and B1.0 catalogs, which together account for approximately $70 \%$ of all asteroid astrometry, are more problematic. We note in particular that the declination maps for these two catalogs are plainly "hot" overall, in agreement with Fig. 4. Also, the RA maps show evidence for hemispheric bias, with a large cold region (negative offset) centered around $45^{\circ} \mathrm{RA}$ extending from pole to pole, and an even stronger hot region opposite, which is, however, roughly limited to the northern hemisphere for A2.0 and the southern hemisphere for B1.0. In contrast, the UCAC and Tycho sky maps show very limited structure, more consistent with random fluctuations.

The procedure followed in this paper is to use the mean catalog differences on each HEALPix cell to debias astrometry from the asssociated catalog in that cell. Specifically, for a given astrometric observation with known catalog, we first compute which of the 49,152 HEALPix sky tiles contains the astrometry and look up the associated RA and DEC offsets, which are subtracted from the raw astrometry to produce debiased astrometry. In light of the foregoing discussion on the low systematic errors seen in the UCAC and Tycho catalogs, we consider that astrometry reduced with these catalogs should not show significant catalog-based systematic bias. Therefore, we apply catalog bias corrections only for astrometry derived from the USNO A1.0, A2.0 and B1.0 catalogs. The catalog comparison file and ancillary documentation are available to other researchers via FTP. ${ }^{4}$

\section{A test on all numbered asteroids}

To evaluate the effectiveness of the debiasing technique outlined in the previous section, we have calculated the orbits of asteroids, both without and with debiasing corrections, to obtain what we call "raw" and "debiased" fits, respectively. We limit the objects considered to those with well-determined orbits, namely the numbered asteroids, to ensure that random errors do not-in the mean-contaminate the results in a significant way. Also, because the objective here is not necessarily to obtain the best possible orbits, but rather to evaluate the efficacy of debiasing, we have used only data that comes within the scope of the debiasing technique, namely astrometry known to be reduced with respect to one of the five catalogs discussed in the previous section. This amounts

\footnotetext{
$4 \mathrm{ftp} / / / \mathrm{ssd}$.jpl.nasa.gov/pub/ssd/debias/debias.tgz.
} 

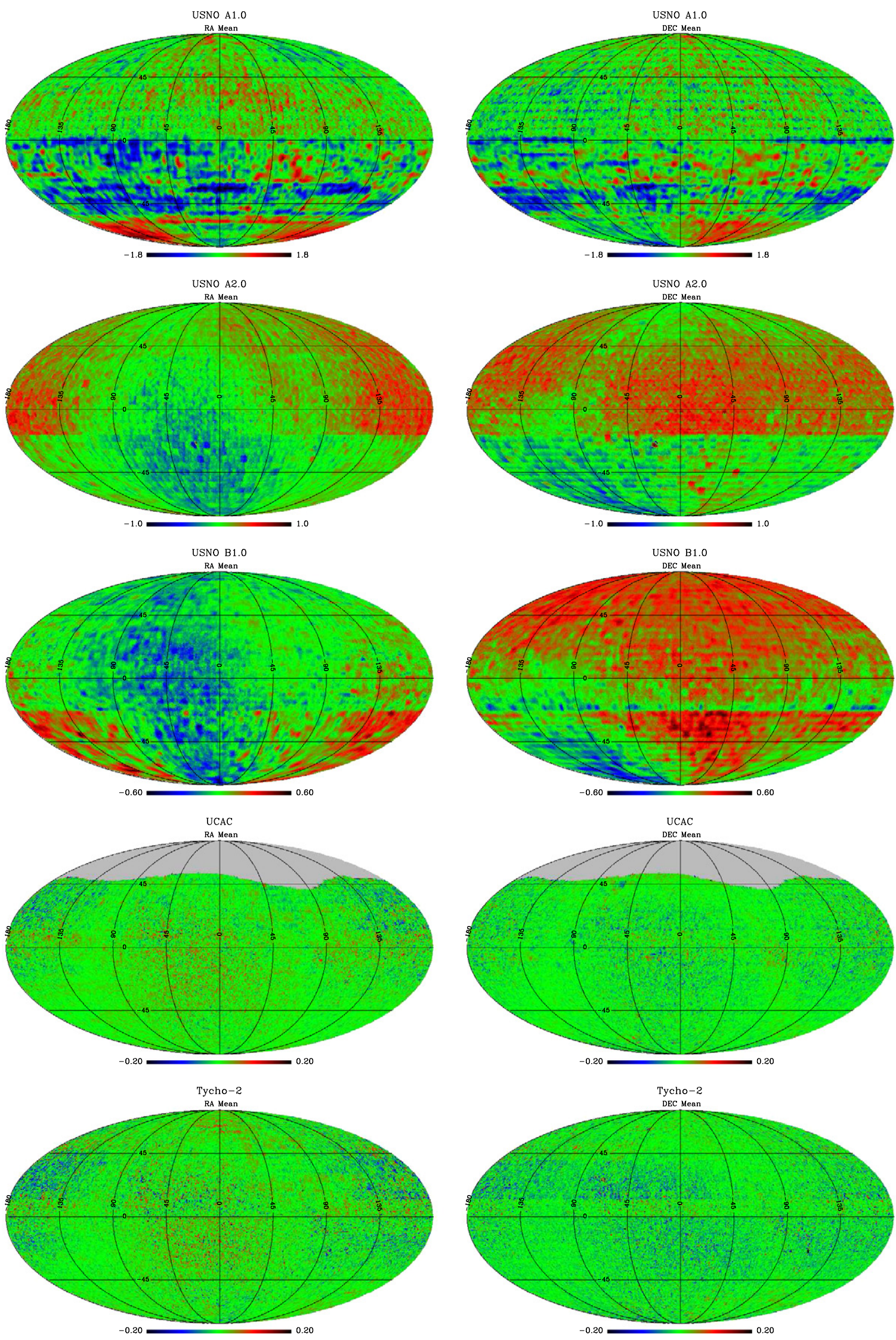

Fig. 5. HEALPix maps of RA and DEC bias with respect to 2MASS for five star catalogs, as described in text. 

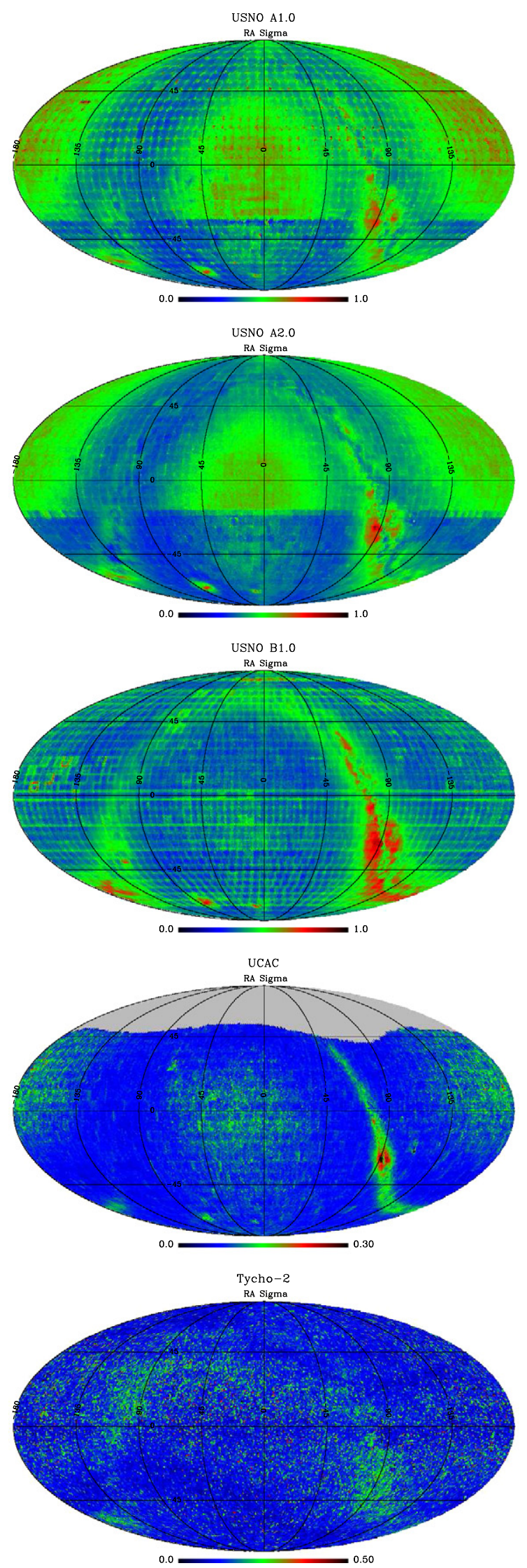

Fig. 6. HEALPix maps of right ascension standard deviation with respect to 2MASS. Declination dispersions are not significantly different. to $48,621,261$ observations of 201,804 asteroids over the interval 1949-11-19 to 2008-12-12. (Note: The 1949-era observations are likely precovery attempts reduced using one of the five catalogs.)

Outlier rejection was used in the orbit calculation, according to the technique described by Carpino et al. (2003), with separate fits for the raw and debiased cases.

The distribution of the change in orbital elements between the raw and debiased fits is depicted in Fig. 7. There we see significant changes in the inclinations of the debiased orbits, relative to their "raw" orbit counterparts. The inclinations generally declined, which is consistent with the fact that the majority of the astrometry falls in the north and has a positive declination bias, which will tend to erroneously increase the estimated inclination. There are smaller changes in the semimajor axis and eccentricity estimates, but still many cases with $>1$ sigma deviations.

\subsection{Correlation of postfit residuals with sky position}

Fig. 8 depicts the postfit residuals for the raw fits, where the sky map reflects the mean postfit residual in each HEALPix cell. As is the case with the asteroids themselves, the majority of numbered asteroid detections are found along the ecliptic. Only cells with at least 10 residual measurements are plotted. For the USNO A1.0, A2.0 and B1.0 catalogs, the raw astrometric residuals show a remarkably similar structure and magnitude to that of the corresponding catalog (Fig. 5). This suggests that, where the observation density is sufficient, the indirect approach to debiasing (by reference to postfit residuals) may have utility in cases where the cata$\log$ bias is poorly constrained.

There is also a significant bias in the postfit residuals of astrometry reduced with the Tycho and UCAC catalogs, despite the low systematic errors seen with these catalogs. This is not particularly surprising, given that the vast majority of observations arise from the significantly biased A2.0 and B1.0 catalogs; these catalogs have skewed the orbit estimates for the raw fits, leading to higher residuals in the unbiased Tycho and UCAC astrometry. In particular, positive declination biases will tend to skew the orbital inclination so that the declination residuals from unbiased observations will also be positive.

The postfit residuals for the debiased orbital fits are depicted in Fig. 9, where the signature seen in Fig. 8 is now substantially reduced, indicating that the biases have been largely eliminated (note, however, the evident striping in the UCAC plots, which we

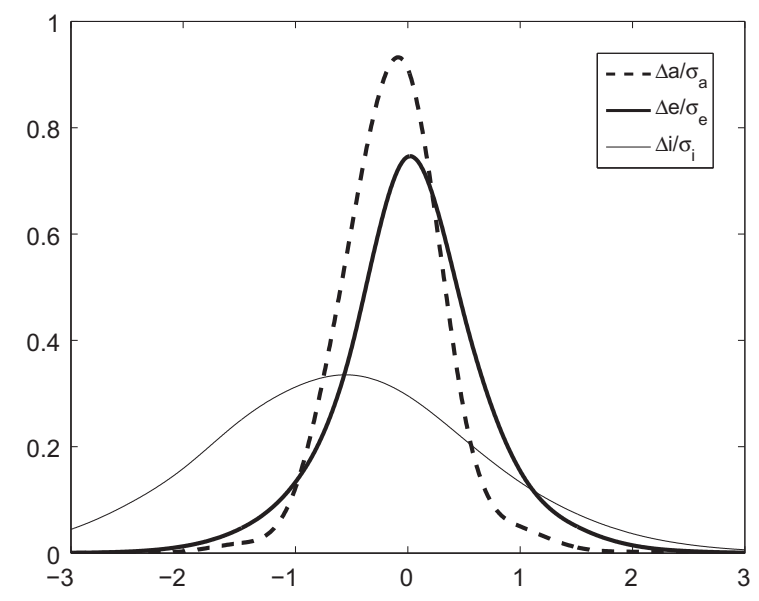

Fig. 7. Distribution of orbital element variation between raw and debiased fits to all numbered asteroids. 

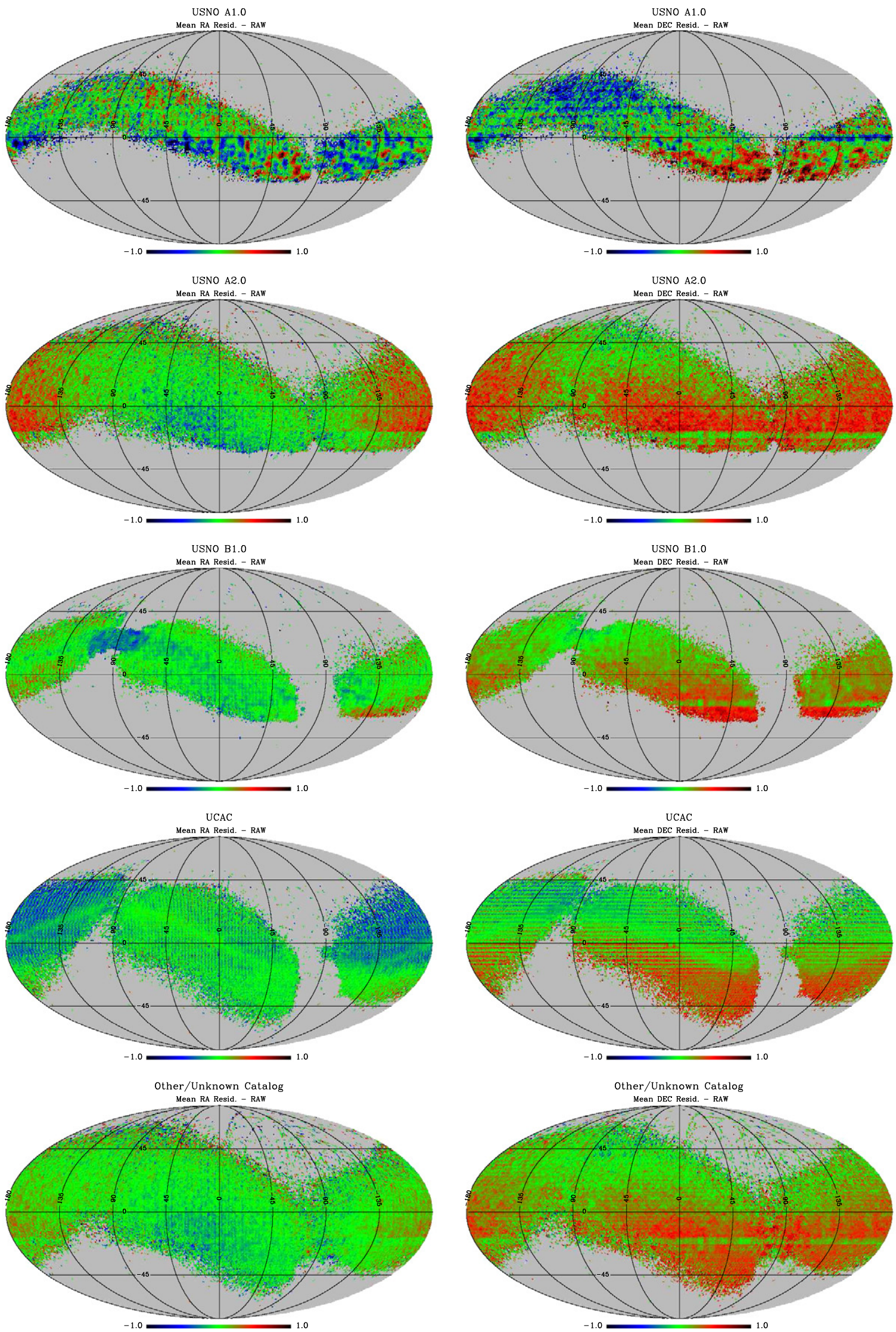

Fig. 8. Mean residuals for fits of raw astrometry to numbered asteroids. The plots depict 49,152 equal area cells using the HEALPix algorithm (Górski et al., 2005). Cells with 10 or fewer observations are not plotted. 

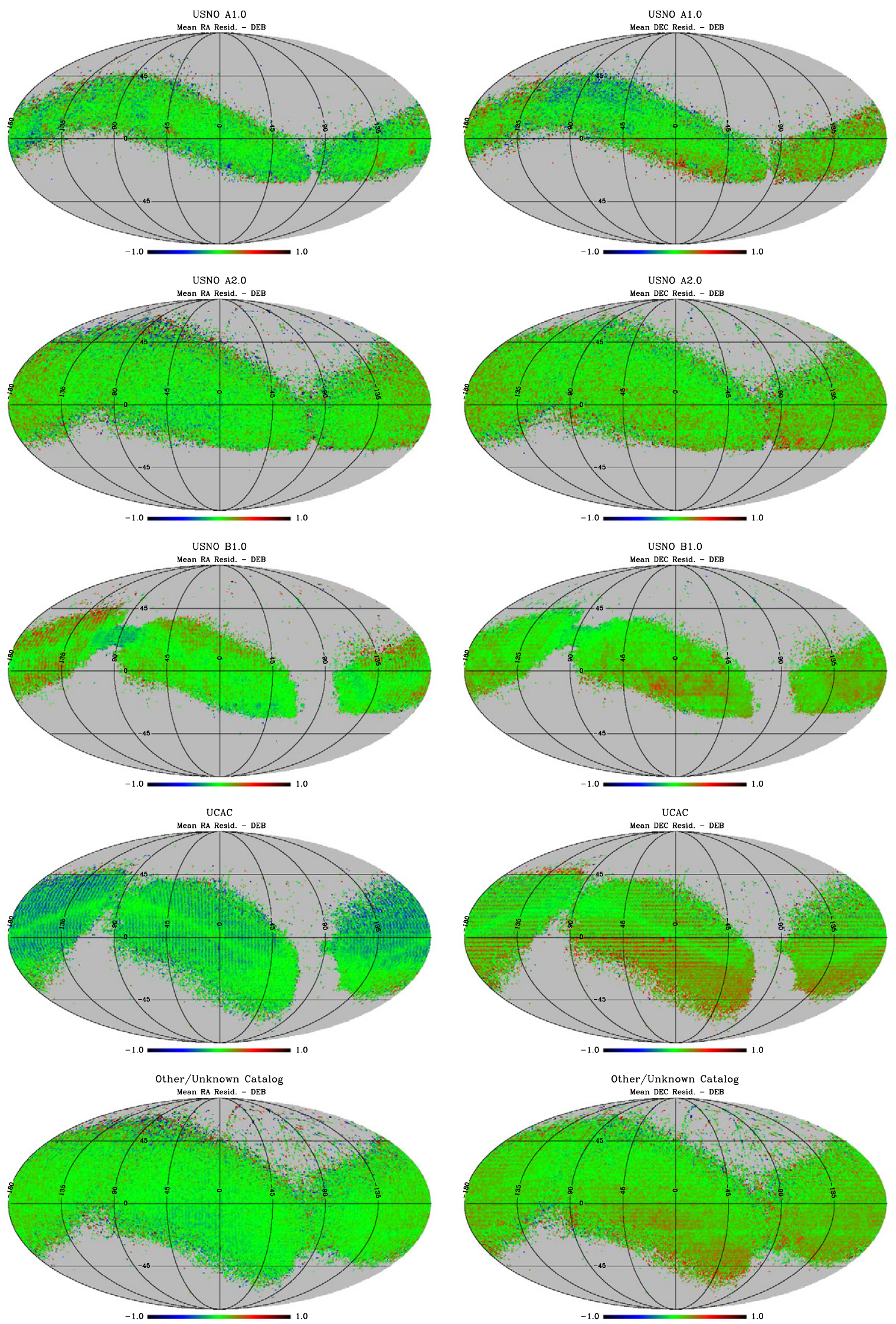

Fig. 9. Mean residuals for fits of debiased astrometry to numbered asteroids, as in Fig. 8. The debiasing has substantially removed the catalog bias signal seen in Fig. 8 . 
USNO A1.0

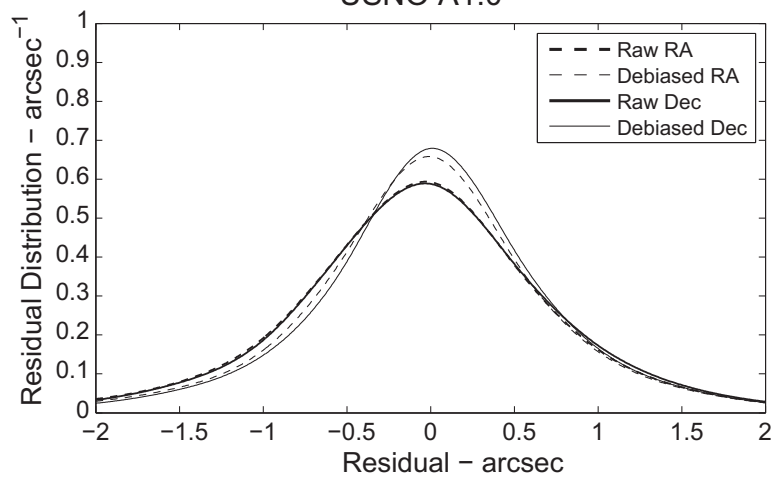

USNO B1.0

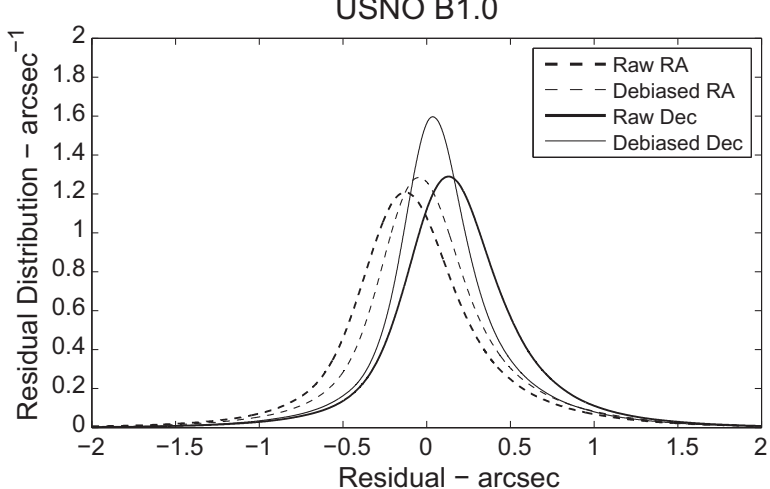

UCAC-2

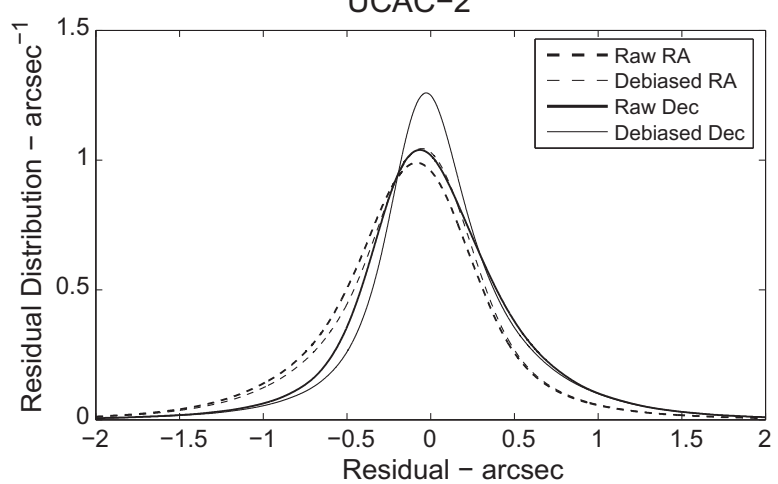

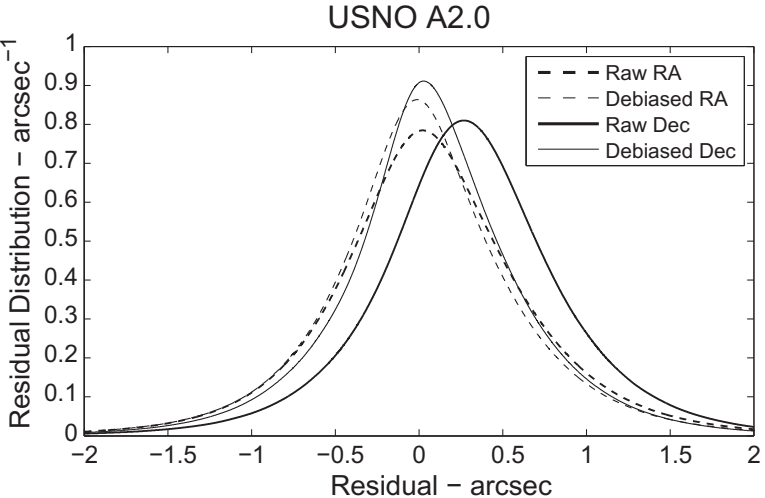

Tycho-2
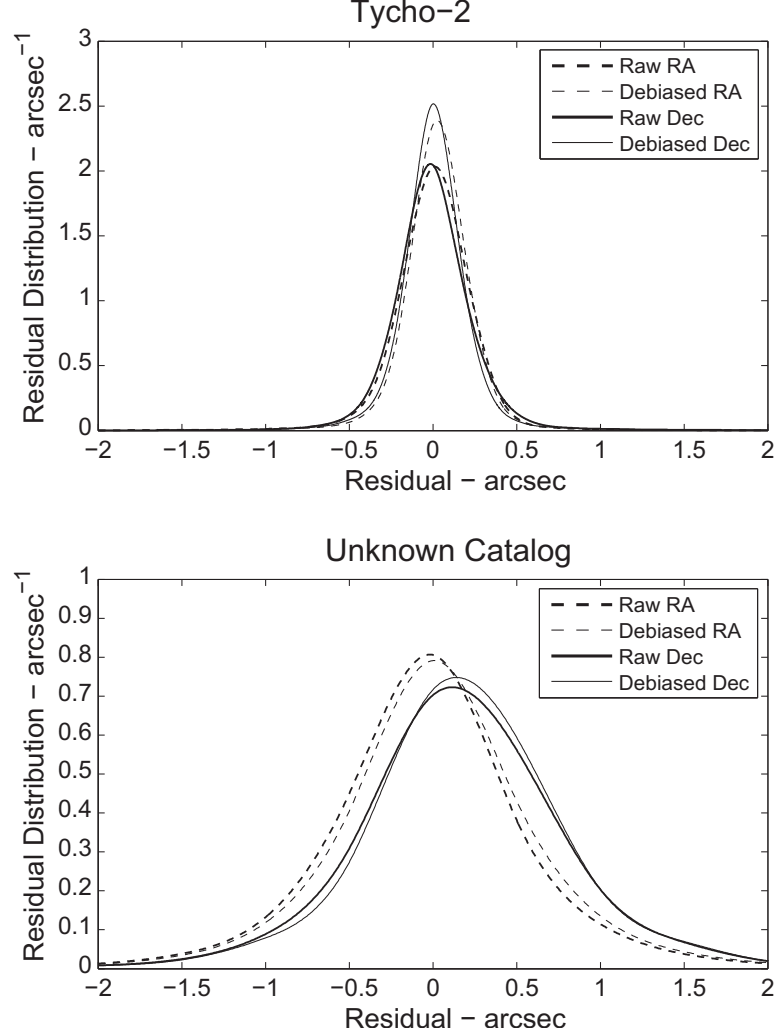

Fig. 10. Distribution of postfit residuals for raw and debiased orbital fits for all numbered asteroids.

will discuss further in Section 5.4). The histograms depicted in Fig. 10 indicate that the debiasing technique does indeed improve the residual distribution; in each case, the curves representing debiased residuals have higher peaks, and are more nearly centered on zero, indicating that the mean residuals and variances have been reduced.

Table 6 quantifies the reduction in mean residuals for each catalog, and also indicates the scatter in the astrometric residuals, before and after debiasing. The table lists the number of observations, separated by catalog and observatory, and the associated RA and DEC residual mean $(\bar{\alpha}, \bar{\delta})$ and standard deviation $(\langle\alpha\rangle,\langle\delta\rangle)$. The ratios between the debiased and raw numbers are also present.

Overall, the star catalog-based debiasing was quite effective, with the mean residuals reduced by as much as $70 \%$; but there are a few curious entries in Table 6 that are worth noting. Of particular interest are the cases that still show a moderate residual bias in the debiased fits. For example, observatory code 703 (Catalina Sky Survey, Mt. Bigelow Schmidt Camera) shows significant RA bias in both the USNO A2.0 and UCAC astrometry; the UCAC DEC bias is rather large, as well. Similarly, E12 (Siding Spring Survey), shows significant DEC bias; and a few other observatories appear to have some signature as well. Also, while the star catalog-based debiasing clearly reduced the mean residuals for observations reduced using USNO B1.0, the mean RA residuals for observatories 699 and 691 are still significant; in effect, their large but opposite biases cancel each other out. We propose a likely explanation for these behaviors below.

\subsection{Untagged astrometry}

Table 6 also details the statistics of the (prefit) residuals associated with observations reduced using unknown catalogs, which appear dominated by observatories 699 (LONEOS) and 644 (NEAT-Haleakala). An examination of the residuals allows us to infer which catalog was used in each case.

Table 7 contains a subset of Table 6 , showing the mean residuals and standard deviations for the five catalogs, as well as the prefit residuals for the unknown observations from 699, 644, 608, 106, 
Table 6

Residual statistics for numbered asteroids by catalog and observatory.

\begin{tabular}{|c|c|c|c|c|c|c|c|c|c|c|c|c|c|c|c|}
\hline \multirow[t]{2}{*}{ Code } & \multirow[t]{2}{*}{ Used } & \multirow[t]{2}{*}{ Percent } & \multirow[t]{2}{*}{ Deleted } & \multicolumn{4}{|c|}{ Debiased (arcsec) } & \multicolumn{4}{|c|}{ Raw (arcsec) } & \multicolumn{4}{|c|}{ Ratio debiased/raw } \\
\hline & & & & $\bar{\alpha}$ & $\langle\alpha\rangle$ & $\bar{\delta}$ & $\langle\delta\rangle$ & $\bar{\alpha}$ & $\langle\alpha\rangle$ & $\bar{\delta}$ & $\langle\delta\rangle$ & $\bar{\alpha}$ & $\langle\alpha\rangle$ & $\bar{\delta}$ & $\langle\delta\rangle$ \\
\hline \multicolumn{16}{|c|}{ USNO A2.0 } \\
\hline ALL & $26,215,118$ & 53.93 & 211,513 & 0.03 & 0.61 & 0.08 & 0.58 & 0.06 & 0.64 & 0.29 & 0.60 & 0.44 & 0.96 & 0.27 & 0.96 \\
\hline 704 & $20,358,631$ & 77.68 & 169,849 & 0.05 & 0.63 & 0.10 & 0.60 & 0.08 & 0.66 & 0.30 & 0.63 & 0.59 & 0.96 & 0.32 & 0.96 \\
\hline 699 & $1,158,767$ & 4.406 & 5,577 & 0.00 & 0.62 & 0.11 & 0.53 & 0.02 & 0.65 & 0.35 & 0.55 & 0.08 & 0.96 & 0.32 & 0.95 \\
\hline 691 & $1,796,063$ & 6.812 & 4,076 & -0.03 & 0.30 & -0.01 & 0.30 & 0.04 & 0.33 & 0.23 & 0.35 & -0.73 & 0.91 & -0.05 & 0.84 \\
\hline 608 & 576,376 & 2.210 & 7,582 & 0.06 & 0.61 & -0.11 & 0.75 & 0.10 & 0.65 & 0.13 & 0.76 & 0.60 & 0.95 & -0.86 & 0.98 \\
\hline 703 & 861,322 & 3.317 & 15,267 & -0.20 & 0.69 & -0.02 & 0.63 & -0.15 & 0.70 & 0.20 & 0.65 & 1.37 & 0.98 & -0.09 & 0.97 \\
\hline 644 & 707,790 & 2.679 & 203 & 0.00 & 0.29 & 0.09 & 0.30 & 0.03 & 0.34 & 0.29 & 0.34 & -0.10 & 0.86 & 0.29 & 0.87 \\
\hline 291 & 222,085 & 0.842 & 359 & -0.10 & 0.46 & 0.04 & 0.32 & -0.03 & 0.46 & 0.28 & 0.36 & 2.83 & 0.98 & 0.14 & 0.88 \\
\hline 599 & 74,867 & 0.285 & 469 & -0.01 & 0.39 & 0.01 & 0.34 & 0.01 & 0.41 & 0.26 & 0.38 & -1.23 & 0.97 & 0.05 & 0.88 \\
\hline 333 & 13,509 & 0.051 & 76 & 0.04 & 0.55 & -0.24 & 0.53 & 0.05 & 0.55 & -0.05 & 0.54 & 0.88 & 0.99 & 4.63 & 0.98 \\
\hline D35 & 29,074 & 0.110 & 16 & 0.11 & 0.39 & 0.02 & 0.38 & 0.20 & 0.41 & 0.21 & 0.40 & 0.53 & 0.95 & 0.09 & 0.94 \\
\hline OTH & 416,634 & 1.607 & 8039 & -0.03 & 0.63 & -0.04 & 0.51 & 0.01 & 0.62 & 0.16 & 0.53 & -1.97 & 1.00 & -0.25 & 0.96 \\
\hline \multicolumn{16}{|c|}{$U C A C 1 \& 2$} \\
\hline ALL & $9,109,348$ & 18.74 & 48,420 & -0.10 & 0.53 & 0.05 & 0.49 & -0.13 & 0.54 & 0.03 & 0.52 & 0.74 & 0.98 & 1.91 & 0.95 \\
\hline 703 & $4,186,043$ & 46.04 & 30,218 & -0.20 & 0.63 & 0.12 & 0.59 & -0.23 & 0.64 & 0.07 & 0.61 & 0.84 & 0.98 & 1.65 & 0.98 \\
\hline G96 & $3,283,638$ & 35.89 & 2,974 & -0.01 & 0.32 & -0.05 & 0.27 & -0.04 & 0.33 & -0.09 & 0.31 & 0.27 & 0.95 & 0.61 & 0.87 \\
\hline E12 & $1,001,493$ & 10.98 & 3,779 & -0.02 & 0.50 & 0.17 & 0.45 & -0.05 & 0.50 & 0.28 & 0.47 & 0.46 & 0.98 & 0.63 & 0.96 \\
\hline 683 & 200,152 & 2.253 & 6,218 & 0.04 & 0.79 & 0.07 & 0.90 & 0.01 & 0.80 & 0.02 & 0.91 & 3.32 & 0.99 & 4.52 & 0.99 \\
\hline J75 & 117,112 & 1.280 & 147 & 0.03 & 0.41 & -0.06 & 0.37 & 0.04 & 0.42 & -0.10 & 0.39 & 0.80 & 0.98 & 0.55 & 0.93 \\
\hline 106 & 92,049 & 1.006 & 44 & 0.02 & 0.40 & -0.05 & 0.39 & -0.02 & 0.42 & -0.14 & 0.41 & -1.01 & 0.97 & 0.33 & 0.95 \\
\hline 143 & 1,443 & 0.016 & 26 & 0.09 & 0.57 & -0.04 & 0.47 & 0.07 & 0.57 & -0.12 & 0.48 & 1.26 & 1.00 & 0.31 & 0.97 \\
\hline OTH & 227,418 & 2.538 & 5014 & 0.01 & 0.51 & -0.00 & 0.42 & -0.03 & 0.51 & -0.04 & 0.45 & -0.26 & 0.99 & 0.02 & 0.94 \\
\hline \multicolumn{16}{|c|}{ USNO B1.0 } \\
\hline ALL & $5,085,749$ & 10.46 & 15,652 & 0.03 & 0.48 & 0.09 & 0.42 & -0.06 & 0.49 & 0.18 & 0.44 & -0.40 & 0.99 & 0.51 & 0.95 \\
\hline 699 & $2,408,525$ & 47.44 & 11,719 & 0.11 & 0.61 & 0.15 & 0.54 & 0.02 & 0.61 & 0.23 & 0.56 & 6.50 & 0.99 & 0.63 & 0.97 \\
\hline 644 & $1,275,002$ & 25.00 & 182 & 0.04 & 0.24 & 0.07 & 0.20 & -0.05 & 0.26 & 0.18 & 0.26 & -0.68 & 0.92 & 0.41 & 0.77 \\
\hline 691 & $1,166,659$ & 22.92 & 2,366 & -0.16 & 0.30 & 0.00 & 0.28 & -0.23 & 0.32 & 0.08 & 0.32 & 0.70 & 0.93 & 0.06 & 0.89 \\
\hline 291 & 63,199 & 1.239 & 12 & -0.22 & 0.39 & 0.07 & 0.26 & -0.29 & 0.40 & 0.15 & 0.29 & 0.76 & 0.96 & 0.49 & 0.89 \\
\hline ОТН & 172,364 & 3.406 & 1373 & 0.05 & 0.48 & 0.03 & 0.39 & -0.04 & 0.46 & 0.14 & 0.41 & -1.15 & 1.02 & 0.24 & 0.94 \\
\hline \multicolumn{16}{|c|}{ USNO A1.0 } \\
\hline ALL & $1,940,649$ & 3.992 & 67,389 & -0.01 & 0.72 & 0.03 & 0.69 & -0.06 & 0.76 & -0.03 & 0.76 & 0.12 & 0.95 & -0.93 & 0.92 \\
\hline 704 & $1,597,775$ & 82.83 & 65,408 & 0.00 & 0.76 & 0.03 & 0.73 & -0.05 & 0.81 & -0.03 & 0.80 & -0.01 & 0.95 & -1.06 & 0.91 \\
\hline 691 & 319,034 & 15.97 & 1594 & -0.06 & 0.49 & 0.01 & 0.46 & -0.12 & 0.51 & -0.06 & 0.48 & 0.47 & 0.96 & -0.22 & 0.97 \\
\hline ОТН & 23,840 & 1.206 & 387 & 0.08 & 0.65 & 0.21 & 0.62 & -0.01 & 0.63 & 0.19 & 0.61 & -5.63 & 1.04 & 1.12 & 1.03 \\
\hline \multicolumn{16}{|c|}{ Tycho-2 } \\
\hline ALL & 220,629 & 0.46 & 564 & 0.03 & 0.24 & 0.01 & 0.25 & -0.01 & 0.26 & 0.00 & 0.28 & -3.85 & 0.91 & -5.38 & 0.89 \\
\hline 689 & 133,287 & 60.26 & 11 & 0.00 & 0.20 & -0.03 & 0.21 & -0.06 & 0.22 & -0.04 & 0.24 & -0.06 & 0.91 & 0.83 & 0.87 \\
\hline OTH & 87,342 & 39.74 & 553 & 0.06 & 0.29 & 0.07 & 0.30 & 0.07 & 0.30 & 0.05 & 0.34 & 0.89 & 0.95 & 1.29 & 0.91 \\
\hline \multicolumn{16}{|c|}{ Unknown } \\
\hline ALL & $5,336,727$ & $\mathrm{~N} / \mathrm{A}$ & 359,619 & 0.02 & 0.73 & 0.16 & 0.68 & -0.07 & 0.73 & 0.13 & 0.70 & -0.31 & 1.00 & 1.23 & 0.98 \\
\hline 699 & $1,180,015$ & 20.84 & 7,153 & 0.11 & 0.68 & 0.31 & 0.57 & 0.03 & 0.67 & 0.27 & 0.60 & 3.65 & 1.02 & 1.14 & 0.96 \\
\hline 644 & $1,119,458$ & 19.85 & 11,277 & 0.04 & 0.51 & 0.43 & 0.51 & -0.04 & 0.49 & 0.40 & 0.52 & -1.06 & 1.04 & 1.08 & 0.97 \\
\hline 608 & 581,208 & 10.43 & 13,175 & 0.00 & 0.78 & 0.12 & 0.80 & -0.09 & 0.77 & 0.11 & 0.80 & -0.02 & 1.02 & 1.07 & 0.99 \\
\hline D29 & 147,281 & 2.591 & 325 & 0.08 & 0.44 & -0.10 & 0.41 & 0.03 & 0.44 & -0.16 & 0.44 & 2.85 & 0.98 & 0.60 & 0.94 \\
\hline 689 & 124,658 & 2.194 & 330 & -0.02 & 0.28 & -0.01 & 0.26 & -0.09 & 0.30 & 0.00 & 0.29 & 0.26 & 0.93 & 1.72 & 0.89 \\
\hline 106 & 28,499 & 0.503 & 172 & 0.03 & 0.54 & 0.26 & 0.47 & -0.06 & 0.52 & 0.20 & 0.50 & -0.55 & 1.02 & 1.29 & 0.94 \\
\hline 300 & 24,032 & 0.430 & 443 & -0.07 & 0.63 & 0.34 & 0.71 & -0.13 & 0.63 & 0.26 & 0.72 & 0.55 & 1.00 & 1.31 & 0.98 \\
\hline ОТН & $2,131,576$ & 43.16 & 326,744 & -0.03 & 0.85 & -0.03 & 0.74 & -0.14 & 0.86 & -0.06 & 0.76 & 0.19 & 0.99 & 0.52 & 0.98 \\
\hline
\end{tabular}


Table 7

Attribution of unknown 699 and 644 observations.

\begin{tabular}{llllll}
\hline Catalog & Code & Mean RA $(\operatorname{arcsec})$ & Sd RA $(\operatorname{arcsec})$ & Mean DEC $(\operatorname{arcsec})$ & Sd DEC $(\operatorname{arcsec})$ \\
\hline USNO A2.0 & ALL & 0.0636 & 0.6367 & 0.2895 & 0.6040 \\
& 699 & 0.0243 & 0.6464 & 0.3470 & 0.5528 \\
& 644 & 0.0292 & 0.3387 & 0.2947 & 0.3428 \\
UCAC2 & ALL & -0.128 & 0.5378 & 0.0279 & 0.5178 \\
USNO B1.0 & ALL & -0.062 & 0.4869 & 0.1799 & 0.4434 \\
& 699 & 0.0176 & 0.6120 & 0.2318 & 0.5565 \\
USNO A1.0 & ALL & -0.063 & 0.2579 & 0.1839 & 0.2562 \\
Tycho-2 & ALL & -0.006 & 0.7644 & -0.028 & 0.7577 \\
Unknown & ALL & -0.070 & 0.2647 & -0.001 & 0.2840 \\
& 699 & 0.0290 & 0.7263 & 0.1296 & 0.6961 \\
& 644 & -0.035 & 0.6704 & 0.2718 & 0.5995 \\
& 608 & -0.085 & 0.4898 & 0.3967 & 0.5215 \\
& 106 & -0.060 & 0.7662 & 0.1077 & 0.8028 \\
\end{tabular}

and 689. The unknown 699 residuals and standard deviations are an excellent match for those of the A2.0 catalog; we feel it is therefore extremely likely that these observations were reduced using A2.0. Similarly, the unknown 644, 608, and 106 statistics are most consistent with A2.0.

As Table 6 indicates, most astrometry reported by 689 is based on the Tycho- 2 star catalog; Table 7 shows that the "unknown" 689 astrometry is perfectly consistent with that catalog, making it likely these observations were also reduced using Tycho-2.

\subsection{Correlation of residuals with time}

As noted in Section 2, the statistical error model proposed by Carpino et al. (2003) requires that the residuals of observations of the same asteroid made by the same observatory have only small correlations that rapidly decay to zero. As Fig. 11 demonstrates, the correlations of the raw residuals were not only significant, but also persisted for several days. In most cases (e.g., observatories G96, 691, and 704), by contrast, the correlations of
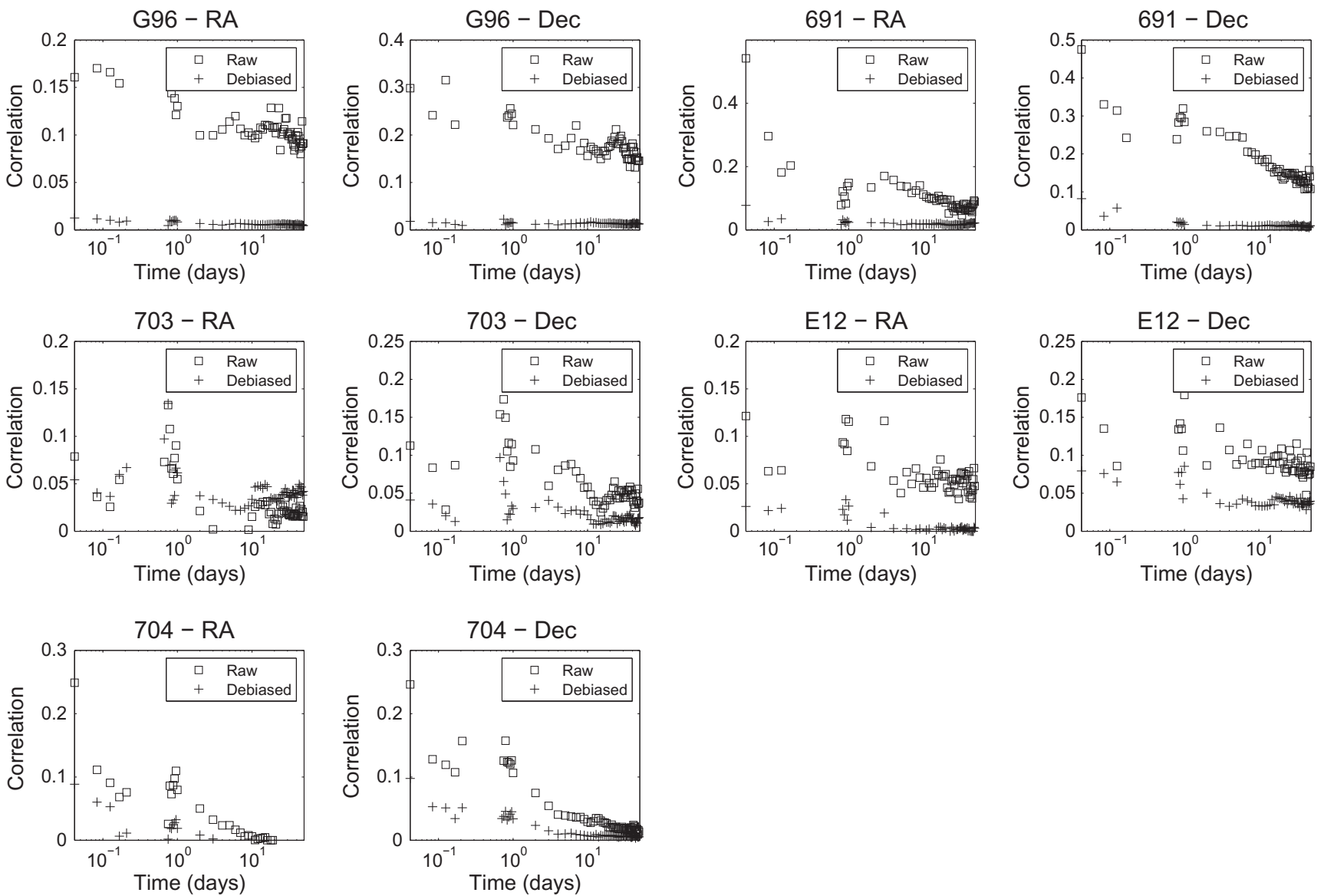

Fig. 11. Correlations between closely-spaced observations of the same asteroid for several large observatories, illustrating the reduction in correlation due to debiasing 
the debiased residuals were consistently smaller, and decayed to insignificance within a day.

However, the debiased correlations for observatories 703 and E12 were not entirely satisfactory; although the debiased correlations were indeed smaller, they did not decay exponentially to zero. And as mentioned above, Table 6 shows the debiased RA and DEC mean residuals for 703 were still significant, as were the debiased DEC mean residuals for E12. This led us to believe another error source existed, unique to these observatories, which might also account for the persistent correlations.

\subsection{Correlation of residuals with focal plane position}

Observatories 703 and E12 both use the UCAC catalog to reduce observations; indeed, the UCAC astrometry set is dominated by these two observatories. Referring to Fig. 9, the debiased UCAC DEC residuals show horizontal striping, while the debiased UCAC RA residuals show vertical striping. We therefore suspected that there were significant errors at the edges of the image field, possibly caused by the failure to account for high-order field distortions in the image reduction process.

To confirm this, we used sky coverage data provided by observatories 703 and E12 to deduce where each asteroid observation lay in the image plane. We then divided the image plane into a $40 \times 40$ grid; and for each individual pixel, we determined the
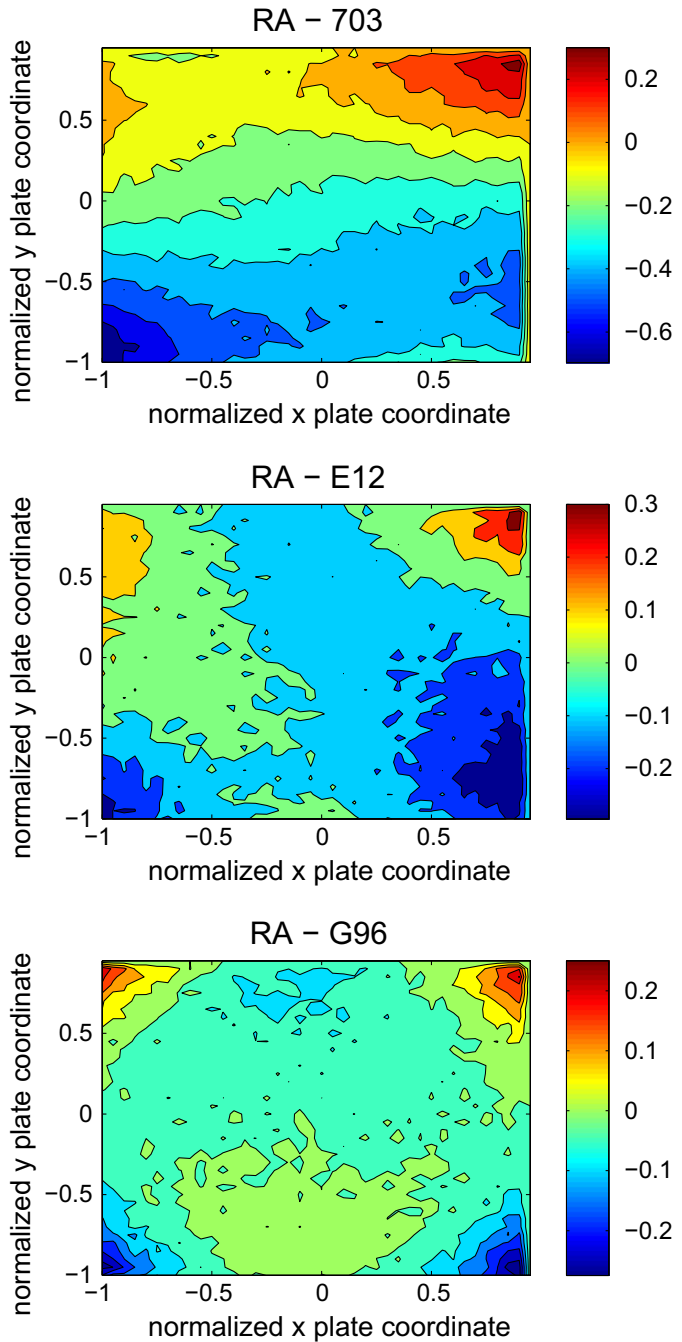

mean debiased RA and DEC residual for all observations lying at that point in the image frame.

As evident in Fig. 12, the debiased residuals for 703 and E12 are generally small near the plate center; but they increase rapidly near the plate boundaries. Similar effects were also observed for observatory G96, which also uses the UCAC catalog.

Further analysis indicated that the field distortion errors for observatory 703 became pronounced between 1998 and 2003; this is consistent with the introduction of new optics in 1999. Since 2003, the plate residuals have been largely stable.

In Table 6 , we note the significant negative value of $\bar{\alpha}$ for code 703 (Mt. Bigelow) with the UCAC catalog, and the similar positive value of $\bar{\delta}$ for E12 (Siding Spring). This may be related to the fact that the two installations use a similar setup, except that the camera is rotated by $90^{\circ}$ at E12 compared to the orientation used at 703 (R. McNaught, private communication). Again, the UCAC astrometry set is dominated by these two observatories; and this persistent effect is evident in the numbered asteroid residual plots for UCAC (Fig. 9), where we can see that a negative residual trend persists in the northern hemisphere RA map, and a positive signal is present in the southern hemisphere DEC map.

\section{Prediction tests}

While the dramatic improvement seen in the astrometric residuals when using the catalog debiasing technique is encouraging, it
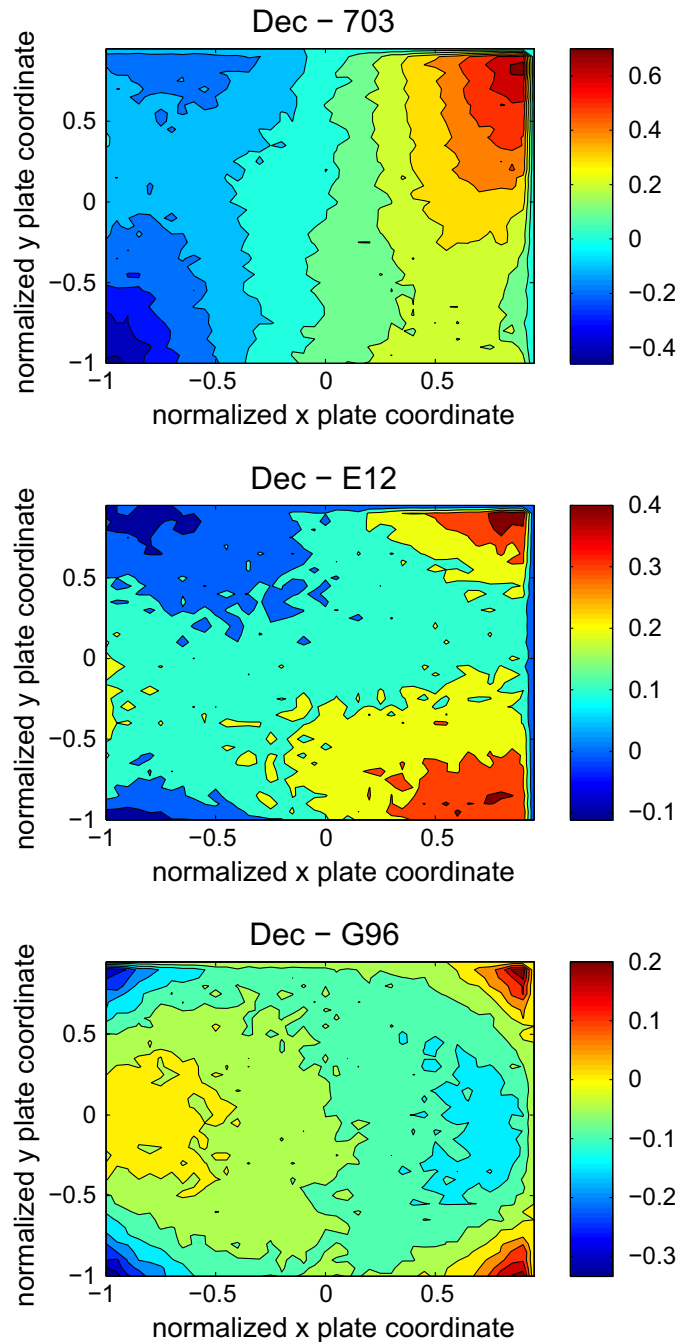

Fig. 12. Mean residuals for the image planes of observatories 703, E12, and G96, illustrating the effects of high order distortions in the image reduction process. 
remains to be seen whether the new orbits are actually superior to those obtained with raw astrometry. Adjusting astrometry with the aim of reducing the postfit residuals can be self-defeating, leaving one with an orbit that appears to be a good fit but does not represent reality. The deciding test is whether or not the new orbits actually predict better or not. To measure prediction performance, we look at how a series of orbital fits to subsets of the entire observational data set compares to a "truth" orbit obtained by fitting over a very long observational arc including all available data.

To compare the prediction performance of the different orbits obtained with raw and debiased astrometry, we have carefully selected a set of main belt asteroids with a thorough CCD observation history. Specifically, we sought asteroids with seven consecutive oppositions, each of which was well-observed with CCD-based astrometry. To start, we selected the 5018 MBAs with absolute magnitude $H$ in the range 14-16 and orbital period in the range 4-6 years. This absolute magnitude translates to diameters from a few to several kilometers, and leads to opposition apparent magnitudes mostly in the range 17-20, faint enough to prevent CCD saturation for most observing setups. The period limit enforces an Earth synodic period between 1.2 and 1.33 years, ensuring that there will have been about 7 oppositions in the previous 10 years.

From among the initial 5018 MBAs, we selected the 222 that actually had observations at seven consecutive oppositions and each opposition with at least 50 CCD observations over at least a 50 day time period. These oppositions generally covered the 2000-2008 time frame. CCD astrometry for which we could not determine the catalog, as described in Section 3 was not considered in the opposition astrometry tallies.

For each of these 222 objects we computed 24 different orbits, that is 12 different observational time spans, each with raw and debiased astrometry. All observations were assigned equal data weights of 1 arcsec. The first of the 12 fit spans, which we use as proxy for the truth orbit, was a fit to all available data, including optical and CCD observations with unknown catalog. These cases, labeled "ALL," used 8-12 oppositions total, with median and mode of nine oppositions. The other 11 fits were based on subsets of the seven consecutive oppositions described above. These fits were intended to reveal the effect of the debiasing technique, and so they used only CCD observations tied to a known catalog. These 11 fits are shown schematically in Fig. 13, where each is shown with its identifying tag that describes the data set used in the respective fit. As an example, for tag "2AS" the "2" indicates that there are two oppositions in the fit, and the " $\mathrm{A}$ " indicates that the two oppositions are after the central (fourth) opposition, which included the prediction epoch. This implies that either oppositions 5 and 6 or 6 and 7 were used in the fit. The final character clarifies this ambiguity: the " $S$ " in the tag indicates that the prediction interval from the
Table 8

Comparison of relative prediction errors for 222 MBAs.

\begin{tabular}{|c|c|c|c|c|}
\hline \multirow[t]{2}{*}{ Case } & & \multicolumn{3}{|c|}{ Relative errors (mean \pm std. dev.) } \\
\hline & & Radial & Transverse & Normal \\
\hline \multirow[t]{2}{*}{$1 \mathrm{BL}$} & Deb. & $0.08 \pm 1.09$ & $0.22 \pm 1.07$ & $0.24 \pm 1.16$ \\
\hline & Raw & $0.08 \pm 1.11$ & $0.27 \pm 1.09$ & $0.76 \pm 1.59$ \\
\hline \multirow[t]{2}{*}{ 1BS } & Deb. & $-0.22 \pm 1.10$ & $0.42 \pm 1.00$ & $0.03 \pm 1.07$ \\
\hline & Raw & $-0.27 \pm 1.17$ & $0.48 \pm 1.05$ & $0.25 \pm 1.33$ \\
\hline \multirow[t]{2}{*}{$1 \mathrm{AS}$} & Deb. & $0.25 \pm 0.73$ & $0.33 \pm 0.68$ & $-0.11 \pm 0.93$ \\
\hline & Raw & $0.28 \pm 0.81$ & $0.38 \pm 0.77$ & $-0.37 \pm 1.64$ \\
\hline \multirow[t]{2}{*}{$1 \mathrm{AL}$} & Deb. & $0.06 \pm 0.86$ & $0.38 \pm 0.83$ & $0.23 \pm 0.80$ \\
\hline & Raw & $0.09 \pm 0.94$ & $0.46 \pm 0.91$ & $0.69 \pm 1.17$ \\
\hline \multirow[t]{2}{*}{$2 \mathrm{BL}$} & Deb. & $-0.01 \pm 1.00$ & $0.10 \pm 0.90$ & $0.26 \pm 1.09$ \\
\hline & Raw & $0.02 \pm 1.13$ & $0.06 \pm 1.05$ & $1.03 \pm 2.50$ \\
\hline \multirow[t]{2}{*}{ 2BS } & Deb. & $-0.06 \pm 1.12$ & $0.16 \pm 1.18$ & $0.52 \pm 1.24$ \\
\hline & Raw & $0.01 \pm 1.19$ & $0.09 \pm 1.25$ & $2.15 \pm 2.48$ \\
\hline \multirow[t]{2}{*}{$2 \mathrm{AS}$} & Deb. & $0.06 \pm 0.85$ & $0.12 \pm 0.82$ & $0.36 \pm 1.27$ \\
\hline & Raw & $0.13 \pm 0.92$ & $0.25 \pm 0.91$ & $1.07 \pm 2.76$ \\
\hline \multirow[t]{2}{*}{$2 \mathrm{AL}$} & Deb. & $0.05 \pm 0.83$ & $0.02 \pm 0.80$ & $0.39 \pm 1.01$ \\
\hline & Raw & $0.13 \pm 0.94$ & $0.09 \pm 0.90$ & $1.20 \pm 1.79$ \\
\hline \multirow[t]{2}{*}{$3 \mathrm{~A}$} & Deb. & $-0.04 \pm 1.12$ & $0.10 \pm 1.08$ & $0.47 \pm 1.07$ \\
\hline & Raw & $0.07 \pm 1.27$ & $-0.01 \pm 1.25$ & $1.84 \pm 1.87$ \\
\hline \multirow[t]{2}{*}{$3 B$} & Deb. & $-0.01 \pm 0.82$ & $0.04 \pm 0.78$ & $0.37 \pm 0.93$ \\
\hline & Raw & $0.05 \pm 0.95$ & $0.15 \pm 0.91$ & $1.10 \pm 1.75$ \\
\hline \multirow[t]{2}{*}{7} & Deb. & $-0.18 \pm 0.62$ & $-0.07 \pm 0.66$ & $-0.02 \pm 0.67$ \\
\hline & Raw & $-0.40 \pm 0.72$ & $-0.12 \pm 0.77$ & $0.26 \pm 0.84$ \\
\hline
\end{tabular}

fit span is short, i.e. oppositions 5 and 6 , as opposed to long ("L"), which would denote oppositions 6 and 7.

We fit orbits to the 12 data sets, using the raw astrometry and then also debiased astrometry. Outlier rejection was accomplished on only two fits, and utilized the method described by Carpino et al. (2003) with a $\chi_{\text {rej }}^{2}=9$. First, the fit for the debiased ALL case allowed for outlier rejection after which the raw ALL fit was obtained with the same data set. Next, the debiased case 7 fit was obtained while allowing for rejection, after which the outlier set was frozen for the 21 subsequent fits for that object. Again, all data weights were 1 arcsec at this point.

For the prediction tests, we propagated each of these 24 orbital fits, for each of the 222 asteroids, to the midpoint of the particular asteroid's fourth opposition, where we mapped the cartesian position and covariance into the classical radial-transverse-normal (RTN) frame positions. (For consistency, we used the debiased case 7 RTN rotation for all the mappings of a given asteroid.) The differences between the orbital predictions for the various cases and that of the "truth" orbit (ALL) in Tables 8 and 9 allow us to determine which statistical treatment provided the better prediction. Of

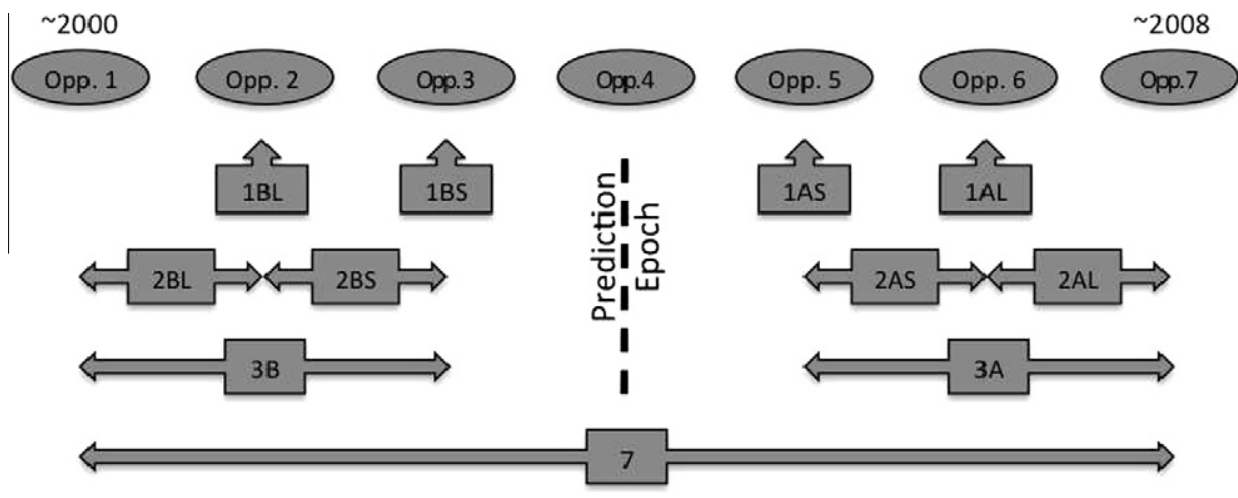

Fig. 13. Schematic diagram depicting the specific oppositions included in the fit spans for the various case labels used in the prediction tests. 
Table 9

Comparison of absolute prediction errors for 222 MBAs.

\begin{tabular}{|c|c|c|c|c|}
\hline \multirow[t]{2}{*}{ Case } & & \multicolumn{3}{|c|}{ Absolute errors (mean \pm std. dev. in kilometers) } \\
\hline & & Radial & Transverse & Normal \\
\hline $1 \mathrm{BL}$ & $\begin{array}{l}\text { Deb. } \\
\text { Raw }\end{array}$ & $\begin{array}{r}1274.48 \pm 29176.26 \\
728.06 \pm 30500.84\end{array}$ & $\begin{array}{l}19614.95 \pm 115110.68 \\
26221.85 \pm 120317.14\end{array}$ & $\begin{array}{r}-2.78 \pm 1590.12 \\
231.44 \pm 1618.86\end{array}$ \\
\hline 1BS & $\begin{array}{l}\text { Deb. } \\
\text { Raw }\end{array}$ & $\begin{array}{l}-2733.96 \pm 61538.02 \\
-5056.61 \pm 58828.86\end{array}$ & $\begin{array}{l}22526.14 \pm 176783.72 \\
26772.96 \pm 162248.03\end{array}$ & $\begin{array}{r}-9.79 \pm 2152.71 \\
42.21 \pm 2292.45\end{array}$ \\
\hline $1 \mathrm{AS}$ & $\begin{array}{l}\text { Deb. } \\
\text { Raw }\end{array}$ & $\begin{array}{l}3673.48 \pm 11653.44 \\
4129.94 \pm 12459.56\end{array}$ & $\begin{array}{l}7868.82 \pm 19486.13 \\
8323.62 \pm 20786.16\end{array}$ & $\begin{array}{r}39.59 \pm 687.46 \\
-16.93 \pm 820.24\end{array}$ \\
\hline $1 \mathrm{AL}$ & $\begin{array}{l}\text { Deb. } \\
\text { Raw }\end{array}$ & $\begin{array}{r}890.40 \pm 13988.90 \\
1397.80 \pm 15375.18\end{array}$ & $\begin{array}{l}19178.37 \pm 51694.49 \\
23802.24 \pm 55250.83\end{array}$ & $\begin{array}{r}72.05 \pm 688.49 \\
198.26 \pm 801.11\end{array}$ \\
\hline 2BL & $\begin{array}{l}\text { Deb. } \\
\text { Raw }\end{array}$ & $\begin{array}{l}-489.47 \pm 6720.73 \\
-220.17 \pm 7197.23\end{array}$ & $\begin{array}{l}3417.92 \pm 25080.41 \\
2199.92 \pm 28812.25\end{array}$ & $\begin{array}{r}43.88 \pm 256.74 \\
189.90 \pm 520.15\end{array}$ \\
\hline 2BS & $\begin{array}{l}\text { Deb. } \\
\text { Raw }\end{array}$ & $\begin{array}{l}-943.50 \pm 7441.94 \\
-498.36 \pm 7766.07\end{array}$ & $\begin{array}{l}3754.07 \pm 23252.53 \\
2797.33 \pm 23545.00\end{array}$ & $\begin{array}{r}77.30 \pm 280.62 \\
376.65 \pm 527.21\end{array}$ \\
\hline $2 \mathrm{AS}$ & $\begin{array}{l}\text { Deb. } \\
\text { Raw }\end{array}$ & $\begin{array}{l}313.42 \pm 3234.76 \\
618.90 \pm 3526.39\end{array}$ & $\begin{array}{r}757.86 \pm 5524.05 \\
1405.35 \pm 6085.32\end{array}$ & $\begin{array}{r}52.67 \pm 220.47 \\
159.60 \pm 465.30\end{array}$ \\
\hline $2 \mathrm{AL}$ & $\begin{array}{l}\text { Deb. } \\
\text { Raw }\end{array}$ & $\begin{array}{l}100.25 \pm 3887.80 \\
438.35 \pm 4510.58\end{array}$ & $\begin{array}{l}1013.54 \pm 14496.75 \\
2120.63 \pm 16640.50\end{array}$ & $\begin{array}{r}77.02 \pm 223.32 \\
227.66 \pm 415.58\end{array}$ \\
\hline $3 \mathrm{~A}$ & $\begin{array}{l}\text { Deb. } \\
\text { Raw }\end{array}$ & $\begin{array}{r}73.93 \pm 1577.52 \\
183.94 \pm 1716.17\end{array}$ & $\begin{array}{l}103.78 \pm 3456.42 \\
391.43 \pm 3707.67\end{array}$ & $\begin{array}{c}54.49 \pm 134.52 \\
156.76 \pm 251.70\end{array}$ \\
\hline $3 B$ & $\begin{array}{l}\text { Deb. } \\
\text { Raw }\end{array}$ & $\begin{array}{r}-223.48 \pm 3921.39 \\
-15.55 \pm 4181.81\end{array}$ & $\begin{array}{r}1004.72 \pm 10900.68 \\
115.73 \pm 11642.35\end{array}$ & $\begin{array}{r}70.24 \pm 183.45 \\
291.84 \pm 310.49\end{array}$ \\
\hline 7 & $\begin{array}{l}\text { Deb. } \\
\text { Raw }\end{array}$ & $\begin{array}{r}-9.39 \pm 32.78 \\
-20.25 \pm 37.33\end{array}$ & $\begin{array}{c}-8.36 \pm 85.96 \\
-14.26 \pm 100.02\end{array}$ & $\begin{array}{l}-3.08 \pm 60.64 \\
22.16 \pm 76.15\end{array}$ \\
\hline
\end{tabular}

course, the prediction errors are statistical, and so the poorer statistical treatment may provide a superior prediction for certain objects. The prediction performance for the ensemble of 222 main belt asteroids is key to reaching a conclusion.

As a typical example, Fig. 14 shows the comparison of the relative prediction errors for fit $2 \mathrm{BL}$. The relative error is the error in sigmas, as given by the $2 \mathrm{BL}$ covariance matrix. In each plot in Fig. 14 there are four curves that compare the raw and debiased 2BL predictions to the raw and debiased ALL orbits. The first three plots in the figure show the 1-D comparison for each of the RTN directions, while the fourth shows the 3-D position error. For reference, the classical $\chi^{2}$ cumulative distribution function, which is expected for an uncorrelated and unbiased Gaussian error distribution, is also plotted.

The results show unambiguously that the debiased astrometry provides predictions that are both more accurate and more in line with the prediction covariance. The greatest improvement is seen in the out-of-plane prediction errors, but there is also a consistent, albeit slight, gain in the radial and transverse directions. There was a possibility of an ambiguous outcome, namely that each statistical method would predict well against its own truth orbit obtained, so that raw would compare well against a raw truth and debiased
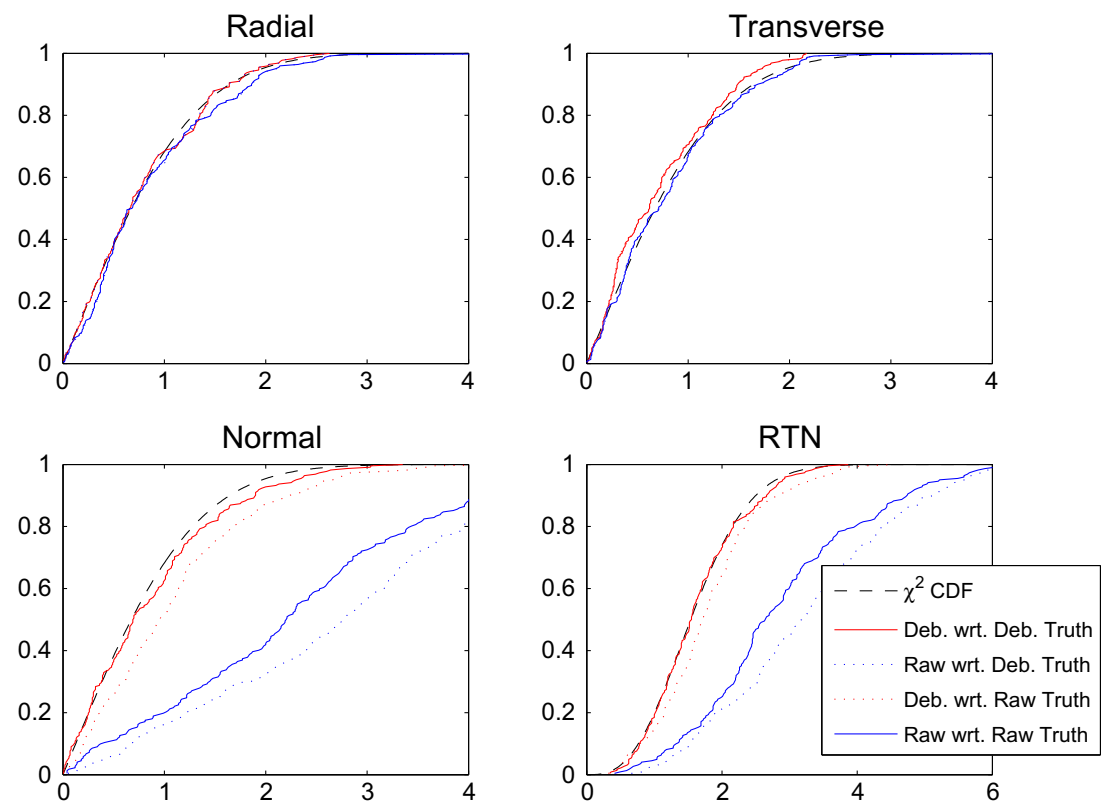

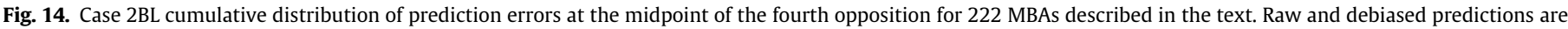
compared to raw and debiased truth orbits obtained using all available astrometry. Abscissae are formal standard deviations, i.e., sigmas. 
would compare well against a debiased truth. As it turns out, and as is clear from Fig. 14, the debiased orbits predict significantly better than the raw orbits no matter which reference orbit is used, and this is true for all 11 cases considered here. As one would expect, there is a slight improvement when comparing the debiased orbit to the debiased truth as opposed to the raw truth, but the salient point is that the debiased orbit outperforms the raw orbit even when both are referenced to the raw truth prediction.
The 3-D comparisons for all 11 fit spans are shown in Fig. 15, where it is clear that the conclusions from the 2BL case carry for every fit span considered. It is important to recognize that these plots depict the relative errors according to the computed prediction uncertainty. The absolute errors depend heavily on the arc length and prediction interval and thus vary substantially among the different cases. For example, the prediction errors, when measured in kilometers, are far greater for 1-opposition fits than
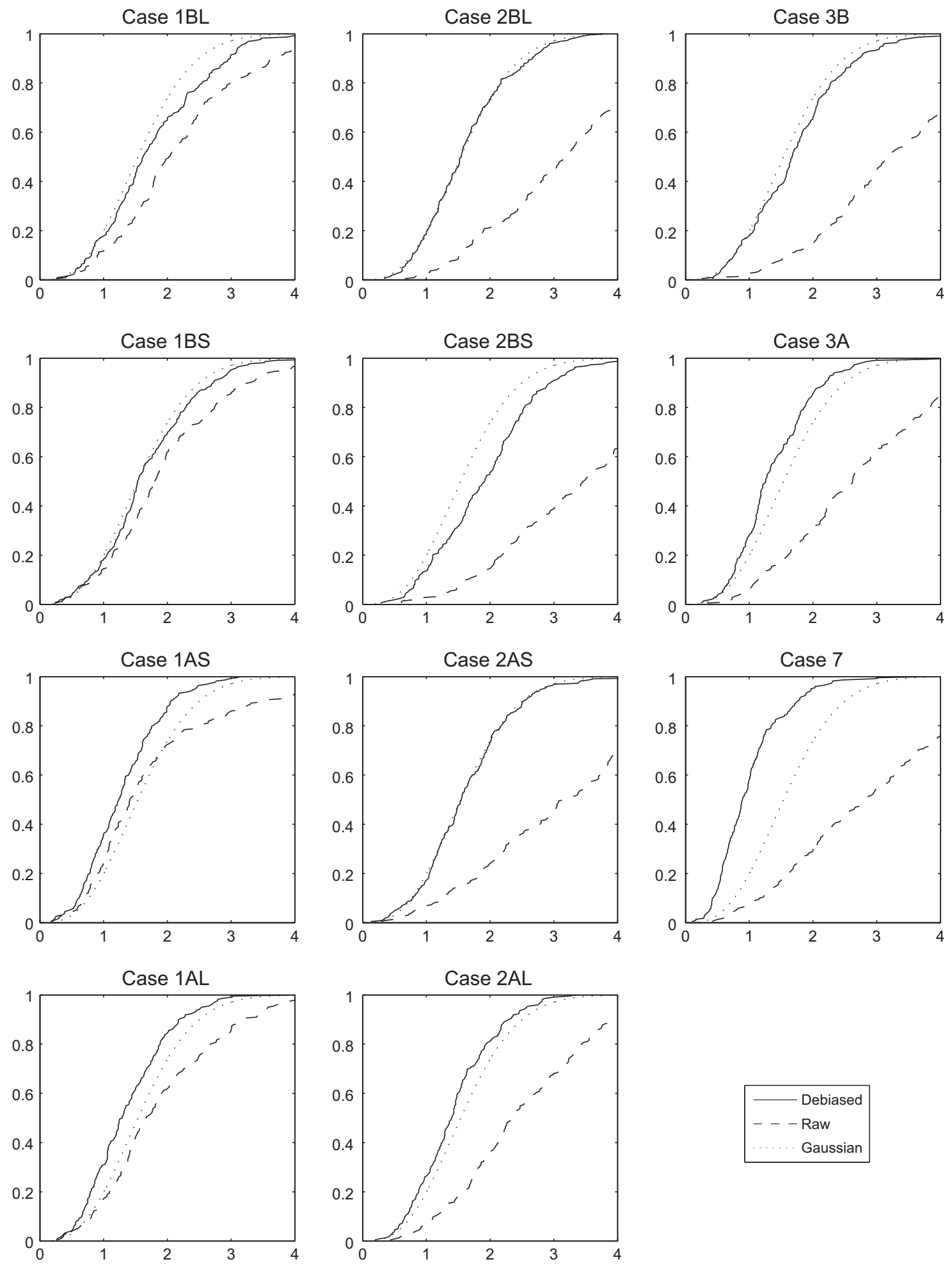

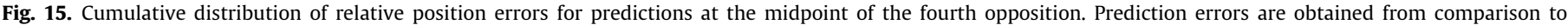
debiased "truth" orbits obtained using all available astrometry. Abscissae in sigmas. 
for 2-opposition fits. Similarly the long predictions have higher absolute errors than short ones for a given data set. However, for a given object, the data sample and weighting were the same for the raw and debiased fits, and so the covariance was the same also.

\subsection{Astrometric weighting}

While the discussion so far has shown emphatically that orbital predictions and postfit residuals are consistently improved with the use of the catalog debiasing technique, some of the debiased CDF curves in Fig. 15 still fall below the $\chi^{2}$ theoretical prediction, suggesting that unresolved biases may be still be present, or-less likely, we believe-that the 1 arcsec weighting is too low. Inspection of the plots suggests that this issue is mostly associated with earlier fitspans and hence earlier data. On the other hand, there are some cases where the debiased CDF is above the theoretical prediction, indicating that the predictions are, in a statistical sense, too good, presumably due to underweighting of the astrometry. This pattern appears to be closely associated to longer arcs and more recent fit spans.

This points to the need for appropriate weighting among the different catalogs and observatories, as opposed to the equal weighting used so far, which has the effect of diminishing the contribution of more accurate observations in favor of less accurate observations. Weighting astrometry according to the observing program, star catalog, epoch of observation, etc. is an obvious place to look for improvement in orbital predictions (Carpino et al., 2003), and with this idea we have tabulated in Table 10 the statistics of the postfit residuals for raw and debiased astrometry for the 7-opposition fits (i.e., case 7).

Among other things, this table affords a comparison of the different catalogs and observing programs, and is analogous to Table 6 , but for the smaller-and carefully selected-set of 222 MBAs. The first conclusion from the table, by now expected, is that the debiased statistics are improved, or show no significant change in the few cases where the raw mean already happened to be very low. Of particular note is the consistent reduction in $\bar{\alpha}$, which had not originally been called out as a problem. We also note that Table 10 is consistent with the analysis in Sections 5.2 and 5.4.

But, for the present purposes, we propose to explore the utility of applying a rudimentary weighting scheme based on the data in Table 10, as opposed to the naive weighting scheme used so far that applies uniform weights to all astrometry. (This usage of "naive" follows Carpino et al. (2003) and should not be construed with its pejorative connotation, but rather in its literal sense of unsophisticated and lacking full information, as is certainly the case when treating all observatories equally.) In an idealized situation, with uncorrelated and unbiased astrometry, the a priori RMS or errors could be inferred from the a posteriori standard deviations of residuals. Despite the fact that our situation still falls short of the ideal, even after catalog debiasing, we will take as weights the tabulated values of $\langle\alpha\rangle$ and $\langle\delta\rangle$ for the debiased fits, later looking at the extent to which this weighting is optimistic. Specifically, we use as weights the standard deviations for each catalog and observatory, or the "OTH" entry for other observatories, as listed on Table 10 . The results from this rudimentary weighting scheme will be compared to that of the naive weighting scheme.

Comparing the prediction performance of the weighted and naive fits is not as simple as comparing the raw and debiased fits because the level of improvement is rather more modest. Moreover, the resulting covariance matrices are significantly different between the naive and weighted fits, and so differences in relative errors may be dominated by the change in covariance. Thus the comparison approach demonstrated in Fig. 14 is inconclusive, because the variations in the weighted and naive orbits are compara- ble to the variations in the weighted and naive truth orbits, and so there is not an adequate truth with which to make the comparison.

Because of this, we take an alternate approach to prediction testing, namely to quantify how well the orbit matches the fourth opposition observations, which were not a part of the associated orbital fit. As an example, case 3B was a fit to oppositions 1-3 for each object. The RMS of prefit residuals obtained for opposition 4 with the case 3B initial conditions is a measure of how well the 3B fit predicted the fourth opposition. Because of the different weighting strategies we do not look at the RMS of weighted residuals, but rather we consider the raw RMS in arcsec, i.e., of unnormalized residuals, to discern which weighting scheme predicts better.

It turns out that there is a clear but modest improvement in the absolute predictions obtained with this rudimentary weighting. Fig. 16 gives the cumulative distributions of the fourth opposition RMS of unweighted residuals. Curves are presented for the RA and DEC RMS, combined and separate, for each of the 11 fitspans. Overall, the DEC predictions are superior to the RA predictions, with the combined RMS falling somewhere in between. This was anticipated because most orbit prediction uncertainty accumulates along track, which maps mostly into RA. The case 7 plot is included although in this case the fourth opposition RMS is postfit and so is not a test of prediction performance. Among the 10 other cases, we see that the weighted distributions are superior to the naive distributions, or they are very close, as is the case for case 2BS. Thus we conclude that the weighting does produce better orbital predictions. This conclusion is reinforced quantitatively by Table 11, which lists the median and 90th percentile combined RMS for each of these ten cases. In every case, the weighted RMS is either significantly less-or substantially the same as-the naive RMS. The table also lists the geometric mean and the median of the ratios between the weighted and naive RMS values, where a number less than one indicates a superior weighted prediction. It is clear again from the table that the weighting produces consistently better predictions. We note that the geometric mean is less than the median in 9 of the 10 cases, implying that for individual objects with a large disparity between the naive and weighted predictions, the results tend to favor the weighted solutions, a conclusion supported by inspection of the raw data.

While we have established that the rudimentary weighting scheme does produce predictions that are superior to the naive scheme, at least in an absolute sense, what is so far missing from the analysis is an understanding of how well the prediction errors match the a posteriori covariance. As one would expect, unresolved biases and correlations cause the standard deviations from Table 11 to be significantly optimistic when translated into weights, and the resulting a posteriori orbit covariances significantly underestimate the orbit estimation errors. To accommodate this we compute and tabulate the safety factor needed to scale the covariance and bring it into line with the orbit errors for each case.

As detailed in Fig. 17, with case $3 \mathrm{~A}$ used as an example, this safety factor was obtained from the ratio of the normalized position errors to that expected from an idealized Gaussian distribution. The values listed in Table 11 were taken at the median, but as is clear from the figure, the value is reasonably constant across the distribution. Six of the values fall within or close to the range 1.5-1.8, while the other four are larger, roughly in the range 2.33.0. It may be significant that these four cases with large safety factors match exactly the four cases that include data from opposition 2 (see Fig. 13). In rough terms, this corresponds to the period from late 2001 to late 2002, which happens to be a time frame when the search efforts were particularly dominated by code 704, the LINEAR search program (Stokes et al., 2000), with approximately $80 \%$ of astrometry obtained by LINEAR. It is also the time frame when the program's second telescope was being brought into operation, which, incidentally, we are not able to distinguish from their 
Table 10

Residual statistics for 222 main belt asteroids by catalog and observatory

\begin{tabular}{|c|c|c|c|c|c|c|c|c|c|c|c|c|c|c|}
\hline \multirow[t]{2}{*}{ Code } & \multirow[t]{2}{*}{ Use/delete } & \multirow[t]{2}{*}{ Percent } & \multicolumn{4}{|c|}{ Debiased (arcsec) } & \multicolumn{4}{|c|}{ Raw (arcsec) } & \multicolumn{4}{|c|}{ Ratio debiased/raw } \\
\hline & & & $\bar{\alpha}$ & $\langle\alpha\rangle$ & $\bar{\delta}$ & $\langle\delta\rangle$ & $\bar{\alpha}$ & $\langle\alpha\rangle$ & $\bar{\delta}$ & $\langle\delta\rangle$ & $\bar{\alpha}$ & $\langle\alpha\rangle$ & $\bar{\delta}$ & $\langle\delta\rangle$ \\
\hline \multicolumn{15}{|c|}{ USNO A2.0 } \\
\hline ALL & $103,471 / 440$ & 68.4 & 0.03 & 0.60 & 0.10 & 0.58 & 0.06 & 0.62 & 0.34 & 0.60 & 0.45 & 0.96 & 0.29 & 0.97 \\
\hline 704 & $85,007 / 380$ & 82.2 & 0.04 & 0.62 & 0.11 & 0.60 & 0.08 & 0.64 & 0.34 & 0.62 & 0.56 & 0.96 & 0.31 & 0.97 \\
\hline 699 & $4343 / 6$ & 4.2 & -0.01 & 0.46 & 0.06 & 0.39 & 0.03 & 0.49 & 0.31 & 0.41 & -0.20 & 0.95 & 0.19 & 0.96 \\
\hline 691 & $3717 / 11$ & 3.6 & -0.06 & 0.31 & 0.01 & 0.34 & 0.00 & 0.33 & 0.25 & 0.38 & 11.99 & 0.95 & 0.05 & 0.89 \\
\hline 608 & $3542 / 13$ & 3.4 & 0.06 & 0.63 & 0.09 & 0.76 & 0.08 & 0.67 & 0.39 & 0.78 & 0.69 & 0.94 & 0.24 & 0.97 \\
\hline 703 & $3343 / 22$ & 3.2 & -0.17 & 0.62 & 0.01 & 0.57 & -0.12 & 0.64 & 0.30 & 0.58 & 1.50 & 0.96 & 0.02 & 0.97 \\
\hline 644 & $1641 / 0$ & 1.6 & -0.03 & 0.24 & 0.11 & 0.28 & 0.00 & 0.31 & 0.37 & 0.30 & 19.67 & 0.77 & 0.30 & 0.92 \\
\hline OTH & $1878 / 8$ & 1.8 & -0.02 & 0.51 & -0.01 & 0.40 & 0.02 & 0.52 & 0.24 & 0.41 & -1.12 & 0.97 & -0.04 & 0.95 \\
\hline \multicolumn{15}{|c|}{$U C A C 1 \& 2$} \\
\hline ALL & $27,211 / 70$ & 18.0 & -0.12 & 0.43 & 0.07 & 0.42 & -0.17 & 0.44 & 0.05 & 0.45 & 0.70 & 0.98 & 1.27 & 0.94 \\
\hline 703 & $12,978 / 37$ & 47.7 & -0.21 & 0.49 & 0.09 & 0.46 & -0.26 & 0.50 & 0.06 & 0.47 & 0.80 & 0.98 & 1.62 & 0.96 \\
\hline G96 & $6787 / 5$ & 24.9 & -0.04 & 0.25 & -0.05 & 0.21 & -0.09 & 0.27 & -0.10 & 0.25 & 0.46 & 0.92 & 0.47 & 0.84 \\
\hline E12 & $4891 / 13$ & 18.0 & -0.03 & 0.41 & 0.17 & 0.43 & -0.07 & 0.42 & 0.27 & 0.44 & 0.36 & 0.99 & 0.62 & 0.98 \\
\hline 683 & $1033 / 13$ & 3.8 & 0.00 & 0.60 & 0.09 & 0.78 & -0.06 & 0.62 & 0.06 & 0.78 & -0.02 & 0.98 & 1.41 & 0.99 \\
\hline OTH & $1522 / 2$ & 5.6 & -0.02 & 0.33 & 0.01 & 0.30 & -0.06 & 0.34 & -0.02 & 0.33 & 0.39 & 0.97 & -0.21 & 0.89 \\
\hline \multicolumn{15}{|c|}{ USNO B1.0 } \\
\hline ALL & $16,056 / 19$ & 10.6 & 0.02 & 0.37 & 0.10 & 0.35 & -0.08 & 0.37 & 0.19 & 0.37 & -0.27 & 0.98 & 0.50 & 0.93 \\
\hline 699 & $9341 / 8$ & 58.2 & 0.09 & 0.42 & 0.14 & 0.40 & -0.01 & 0.42 & 0.25 & 0.42 & -8.31 & 0.99 & 0.56 & 0.96 \\
\hline 644 & $4092 / 0$ & 25.5 & -0.02 & 0.18 & 0.04 & 0.17 & -0.13 & 0.20 & 0.14 & 0.23 & 0.18 & 0.90 & 0.30 & 0.71 \\
\hline 691 & $2165 / 6$ & 13.5 & -0.20 & 0.25 & 0.02 & 0.28 & -0.30 & 0.26 & 0.07 & 0.31 & 0.66 & 0.94 & 0.27 & 0.91 \\
\hline OTH & $458 / 5$ & 2.9 & -0.01 & 0.49 & 0.04 & 0.40 & -0.10 & 0.50 & 0.15 & 0.43 & 0.05 & 0.99 & 0.29 & 0.94 \\
\hline \multicolumn{15}{|c|}{ GSC-ACT } \\
\hline ALL & $1628 / 19$ & 1.1 & 0.06 & 0.55 & 0.12 & 0.56 & -0.04 & 0.55 & 0.10 & 0.58 & -1.52 & 1.01 & 1.15 & 0.97 \\
\hline 333 & $1023 / 5$ & 62.8 & 0.09 & 0.52 & 0.06 & 0.46 & -0.05 & 0.52 & 0.06 & 0.49 & -1.87 & 1.00 & 1.07 & 0.93 \\
\hline OTH & $605 / 14$ & 37.2 & 0.02 & 0.60 & 0.21 & 0.70 & -0.03 & 0.59 & 0.18 & 0.71 & -0.53 & 1.01 & 1.20 & 0.99 \\
\hline \multicolumn{15}{|c|}{ USNO A1.0 } \\
\hline ALL & $1315 / 10$ & 0.9 & -0.02 & 0.58 & 0.01 & 0.51 & -0.11 & 0.62 & -0.05 & 0.57 & 0.21 & 0.94 & -0.20 & 0.89 \\
\hline 704 & $1103 / 10$ & 83.9 & 0.00 & 0.59 & 0.00 & 0.52 & -0.08 & 0.63 & -0.06 & 0.59 & 0.00 & 0.93 & 0.07 & 0.88 \\
\hline ОТН & $212 / 0$ & 16.1 & -0.14 & 0.55 & 0.09 & 0.47 & -0.25 & 0.55 & -0.02 & 0.46 & 0.59 & 0.99 & -4.26 & 1.03 \\
\hline \multicolumn{15}{|c|}{ CMC } \\
\hline \multicolumn{14}{|c|}{ Tycho-2 } & 0.96 \\
\hline ALL & $459 / 5$ & 0.3 & 0.04 & 0.40 & -0.04 & 0.40 & -0.04 & 0.42 & -0.01 & 0.42 & -0.81 & 0.95 & 2.47 & 0.94 \\
\hline 689 & $370 / 0$ & 80.6 & 0.01 & 0.26 & -0.04 & 0.31 & -0.07 & 0.28 & -0.01 & 0.36 & -0.20 & 0.91 & 4.38 & 0.88 \\
\hline ОТН & $89 / 5$ & 19.4 & 0.12 & 0.72 & -0.04 & 0.63 & 0.05 & 0.75 & -0.04 & 0.62 & 2.32 & 0.97 & 0.98 & 1.02 \\
\hline \multicolumn{14}{|l|}{ GSC 1} & 0.97 \\
\hline \multicolumn{15}{|c|}{ Unknown } \\
\hline ALL & 9515 & $\mathrm{~N} / \mathrm{A}$ & 0.07 & 0.58 & 0.34 & 0.59 & -0.03 & 0.57 & 0.31 & 0.59 & -2.46 & 1.03 & 1.13 & 0.99 \\
\hline 699 & 3090 & 32.5 & 0.05 & 0.56 & 0.44 & 0.41 & -0.04 & 0.53 & 0.40 & 0.41 & -1.23 & 1.04 & 1.10 & 0.99 \\
\hline 644 & 2645 & 27.8 & 0.09 & 0.43 & 0.41 & 0.49 & -0.01 & 0.40 & 0.36 & 0.50 & -8.47 & 1.08 & 1.13 & 0.98 \\
\hline 608 & 2049 & 21.5 & 0.13 & 0.77 & 0.31 & 0.85 & 0.02 & 0.76 & 0.29 & 0.84 & 7.28 & 1.01 & 1.05 & 1.00 \\
\hline D29 & 484 & 5.1 & 0.03 & 0.36 & -0.05 & 0.32 & -0.03 & 0.37 & -0.10 & 0.34 & -1.13 & 0.97 & 0.46 & 0.95 \\
\hline 689 & 206 & 2.2 & -0.02 & 0.23 & -0.01 & 0.29 & -0.07 & 0.26 & -0.05 & 0.33 & 0.32 & 0.90 & 0.18 & 0.86 \\
\hline 106 & 178 & 1.9 & 0.03 & 0.56 & 0.33 & 0.51 & -0.06 & 0.55 & 0.28 & 0.52 & -0.55 & 1.02 & 1.18 & 0.98 \\
\hline 300 & 177 & 1.9 & -0.07 & 0.60 & 0.28 & 0.69 & -0.14 & 0.61 & 0.24 & 0.71 & 0.51 & 1.00 & 1.18 & 0.97 \\
\hline
\end{tabular}


Case 1BL

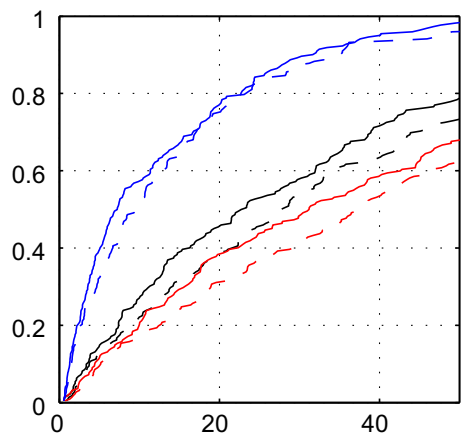

Case 1BS

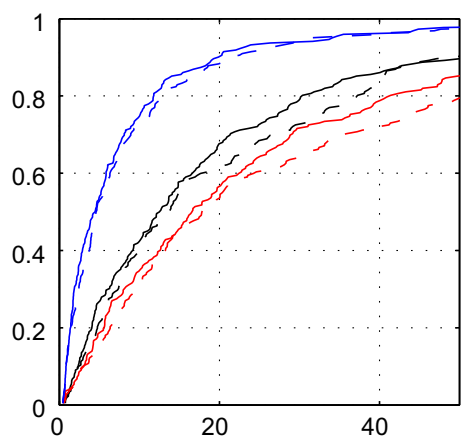

Case $1 \mathrm{AS}$

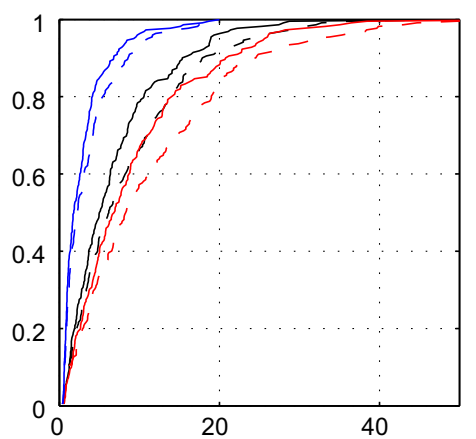

Case $1 \mathrm{AL}$

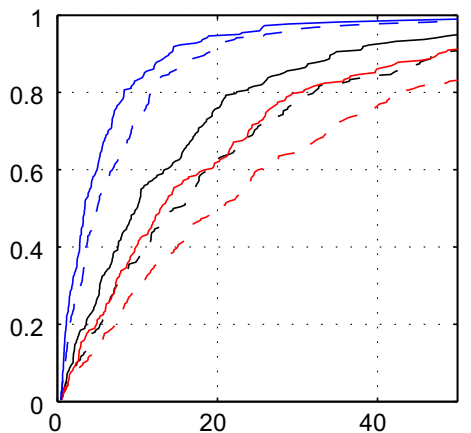

Case 2BL

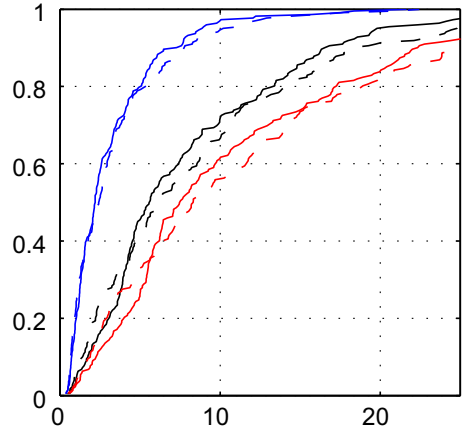

Case 2BS

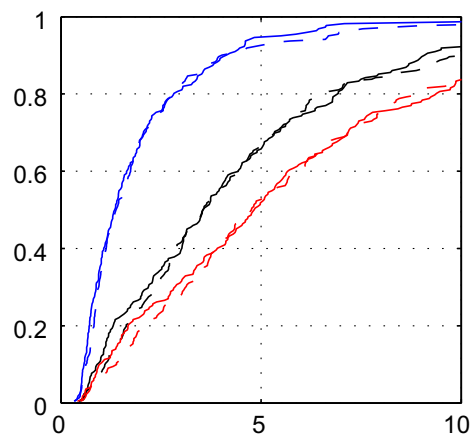

Case 2AS

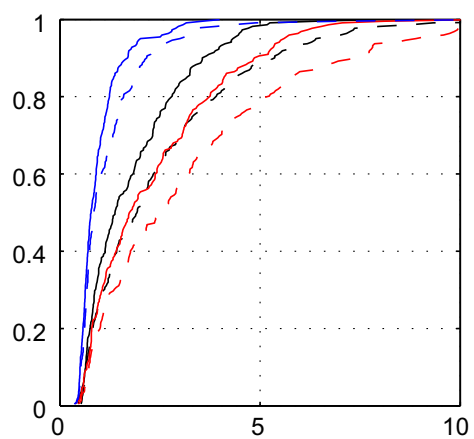

Case 2AL

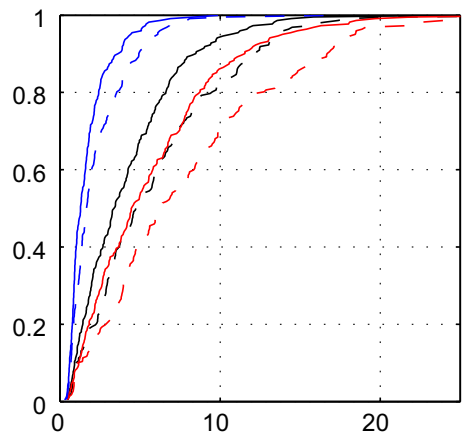

Case 3B

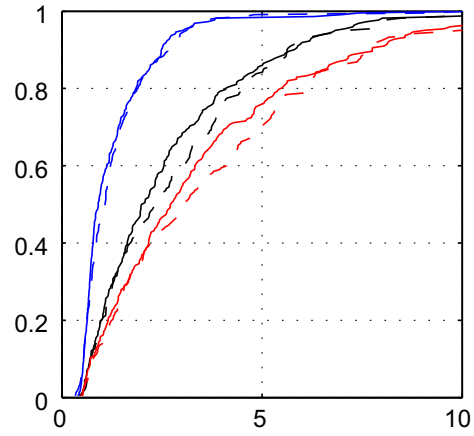

Case $3 \mathrm{~A}$

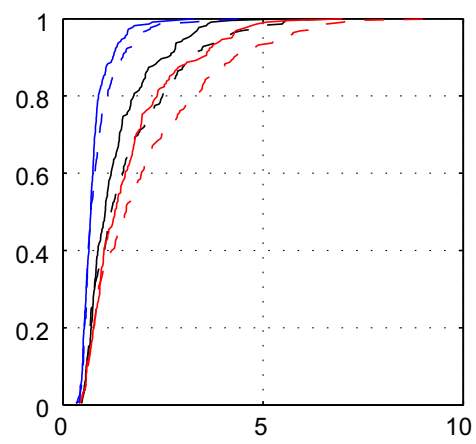

Case 7

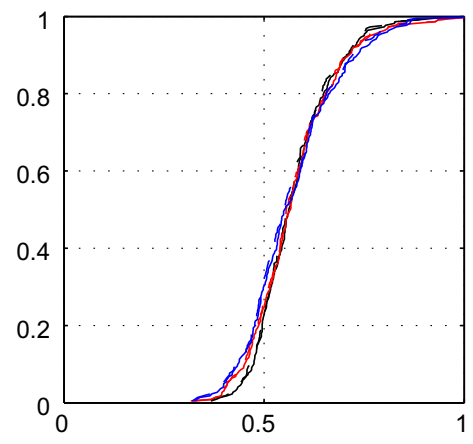

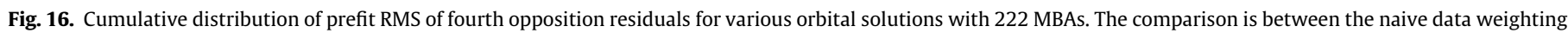

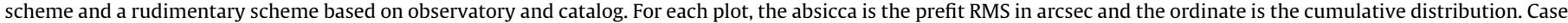
7 is included for reference, although only postfit residuals are available for the fourth opposition.

first telescope by reference to the astrometric data delivered by the MPC. In any case, the mean of the tabulated safety factors is $2.03 \pm 0.56$. From all of this we conclude that scaling the a posteriori standard deviation of residuals by a factor of 2 , and using these as weights leads to improved absolute and relative predictions, at least for the present ensemble of 222 MBAs.

\section{Applications}

\subsection{Asteroid mass estimation}

Careful asteroid orbit determination affords the possibility of observing the perturbation by many of the larger main belt 
Table 11

Comparison of prefit RMS of fourth opposition residuals, with and without observatory-specific weighting, for 222 MBAs.

\begin{tabular}{|c|c|c|c|c|c|c|c|}
\hline \multirow[t]{2}{*}{ Case } & \multicolumn{2}{|c|}{ Ratio weighted/naive } & \multicolumn{2}{|c|}{ Median (arcsec) } & \multicolumn{2}{|c|}{ 90th percentile (arcsec) } & \multirow[t]{2}{*}{ Safety factor } \\
\hline & Geom. mean & Median & Naive & Weighted & Naive & Weighted & \\
\hline $1 \mathrm{AL}$ & 0.715 & 0.729 & 14.979 & 10.014 & 48.179 & 34.651 & 1.51 \\
\hline $1 \mathrm{AS}$ & 0.835 & 0.839 & 6.181 & 5.076 & 18.042 & 15.558 & 1.49 \\
\hline $1 \mathrm{BS}$ & 0.884 & 0.949 & 13.591 & 12.597 & 49.820 & 52.295 & 1.82 \\
\hline $1 \mathrm{BL}$ & 0.834 & 0.890 & 27.926 & 23.061 & 80.177 & 74.104 & 2.29 \\
\hline $2 \mathrm{AL}$ & 0.726 & 0.736 & 4.748 & 3.403 & 12.275 & 8.495 & 1.59 \\
\hline 2AS & 0.780 & 0.818 & 1.928 & 1.379 & 5.367 & 3.589 & 1.79 \\
\hline 2BS & 0.936 & 0.947 & 3.557 & 3.565 & 10.168 & 9.112 & 2.97 \\
\hline $2 \mathrm{BL}$ & 1.008 & 0.964 & 6.186 & 5.600 & 18.514 & 16.634 & 2.65 \\
\hline $3 \mathrm{~A}$ & 0.862 & 0.920 & 1.207 & 1.059 & 3.227 & 2.644 & 1.54 \\
\hline $3 B$ & 0.936 & 0.969 & 2.324 & 2.026 & 5.637 & 5.827 & 2.66 \\
\hline
\end{tabular}

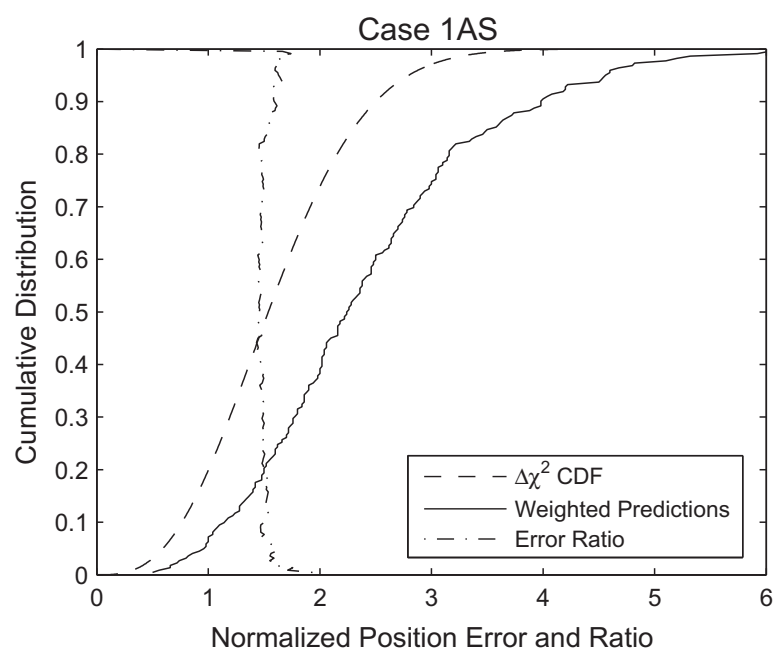

Fig. 17. Cumulative distribution of normalized position errors for weighted fits to case $3 \mathrm{~A}$ (as an example), depicted with the $\Delta \chi^{2} \mathrm{CDF}$. The ratio between the actual and ideal errors at a given cumulative distribution level is also depicted. This is the scale factor required to bring the weighted a posteriori covariance into agreement with the Gaussian distribution.

asteroids on some of the more numerous smaller asteroids, termed test particles in this context. The combination of the measured perturbation, usually an offset in orbital longitude, and the estimated approach circumstances leads to an estimate of the mass of the larger asteroid. However, the mass estimate can be skewed if the asteroid orbit determinations are subject to catalog bias.

Aside from events associated with 1 Ceres or 4 Vesta, most asteroid mass determination events thus far discovered involve cumulative deflections on the order of a few arcsec (or less). Since the debiasing corrections are non-trivial on this scale, we expected some improvement in mass determination relative to the use of raw observations.

Having said that, successful mass determination encounters usually involve changes in an asteroid's semimajor axis (and thus its mean motion), resulting in cumulative along-track shifts in sky position versus the pre-encounter trajectory. As we saw in Fig. 7, however, while debiasing does indeed improve determination of the semimajor axis, the improvement is somewhat less pronounced than for the eccentricity or inclination. Therefore, it seemed likely that the improvement in mass determination would be relatively modest.

Table 12 illustrates the results of 11 such comparative trials, representing all known mass determination events (aside from 1 Ceres or 4 Vesta) with encounters after 1997, and with a significance (defined as the ratio of mass to mass uncertainty) greater than 2.5. The "best" mass values (representing published values) were calculated using the full set of available observations; while the "debiased" and "raw" masses were calculated using only post-1995 observations (since observations before this time precede availability of the USNO catalogs, and thus cannot be debiased). Consequently, the "best" masses benefit from a far better knowledge of the test asteroid's pre-encounter trajectory. However, our intent here is not to obtain the best possible mass estimates, but rather to determine if debiasing results in superior values.

We frankly believe that the formal uncertainties in asteroid mass determination likely understate the actual effects of unmodeled perturbations and unknown error sources; so while we have listed the best currently available mass for each asteroid (with no debiasing applied), these values may not be sufficiently well determined to act as absolute references for comparison. We have therefore also provided estimates of bulk density and porosity; clearly unrealistic values should act as an additional check in these comparisons.

In 10 of the 11 cases, the debiased mass is closer to the best currently available value than the mass derived from raw observations. The exception being the case of $10 / 24,433$, where the raw and debiased results are virtually identical. This advantage is most dramatically apparent in the case of $9 / 29,818$, where the difference between the debiased and raw masses is on the order of ten times the formal uncertainty (i.e., $10-\sigma$ ). Indeed, one might argue that the debiased mass for 9 Metis, indicating a near-zero bulk porosity, is more realistic than the best currently available value, which results in a negative porosity, which we note again is derived with raw astrometry.

The apparent advantage of using debiased observations is still evident, although not as starkly, in the results for $1 / 13,801,7 /$ $52,443,10 / 3946,15 / 50,278$, and $189 / 6224$. In each case, not only is the debiased mass closer to the best available value, but the resulting bulk porosity is equally (if not more) reasonable. In the cases of $3 / 17,715,6 / 4497$ and $10 / 57,493$, the formal uncertainty in the calculated mass is larger than the mass itself; nevertheless, we believe it is significant that the calculated masses are consistently closer to the best available values, and the resulting bulk porosities are more reasonable than those resulting from raw observations.

\subsection{Impact hazard assessment}

From a public perspective, one of the most notable applications of asteroid orbit determination is the assessment of the impact risk posed by potentially hazardous asteroids. A natural question is to what extent the debiasing techniques proposed in this paper will affect such analyses. There are two independent, automated impact-monitoring systems, Sentry ${ }^{5}$ at NASA's Jet Propulsion

\footnotetext{
5 http://neo.jpl.nasa.gov.
} 
Table 12

Mass/porosity determinations using raw and debiased observations.

\begin{tabular}{|c|c|c|c|c|c|c|c|c|c|c|}
\hline Method & Subject Ast. & Radius (km) & Test ast. & Arc & $N_{o b s}$ & Enc. yr. & Mass $\left(M_{\odot}\right)$ & Rel. Diff. ${ }^{a}$ & $\begin{array}{l}\text { Bulk density } \\
(\mathrm{g} / \mathrm{cm})^{\mathrm{e}}\end{array}$ & Bulk porosity ${ }^{b}$ \\
\hline Raw & 1 Ceres & $476.2 \pm 3.6^{d}$ & 13801 & 1997.8-2008.9 & 451 & 1998.1 & $4.42 \pm 1.16 \times 10^{-10}$ & & $1.94 \pm 0.51$ & $0.43 \pm 0.15$ \\
\hline Debiased & & & & $1997.8-2008.9$ & 451 & 1998.1 & $4.76 \pm 1.16 \times 10^{-10}$ & 0.29 & $2.09 \pm 0.51$ & $0.38 \pm 0.15$ \\
\hline Best $^{\mathrm{c}}$ & & & & & & & $4.753 \pm 0.007 \times 10^{-10}$ & & $2.09 \pm 0.05$ & $0.39 \pm 0.01$ \\
\hline Raw & 3 Juno & $129 \pm 3^{\mathrm{e}, \mathrm{f}}$ & 17715 & 1997.9-2008.9 & 497 & 2000.8 & $2.71 \pm 1.99 \times 10^{-11}$ & & $6.02 \pm 4.44$ & $-0.77 \pm 1.31$ \\
\hline Debiased & & & & 1997.9-2008.9 & 497 & 2000.8 & $1.46 \pm 1.98 \times 10^{-11}$ & -0.63 & $3.25 \pm 4.40$ & $0.05 \pm 1.30$ \\
\hline Best $^{\mathrm{C}}$ & & & & & & & $1.51 \pm 0.03 \times 10^{-11}$ & & $3.36 \pm 0.24$ & $0.01 \pm 0.07$ \\
\hline Raw & 6 Hebe & $93 \pm 1.45^{\mathrm{h}, \mathrm{i}}$ & 4497 & 1997.1-2008.5 & 654 & 1997.5 & $22.30 \pm 25.30 \times 10^{-12}$ & & $13.17 \pm 14.95$ & $-2.87 \pm 4.40$ \\
\hline Debiased & & & & $1997.1-2008.5$ & 654 & 1997.5 & $7.20 \pm 25.20 \times 10^{-12}$ & -0.60 & $4.25 \pm 14.88$ & $-0.25 \pm 4.38$ \\
\hline Best $^{\mathrm{g}}$ & & & & & & & $6.46 \pm 0.32 \times 10^{-12}$ & & $3.81 \pm 0.26$ & $-0.12 \pm 0.08$ \\
\hline Raw & 7 Iris & $106 \pm 5^{\mathrm{f}, \mathrm{h}}$ & 52443 & 1998.8-2008.6 & 396 & 2001.2 & $12.1 \pm 5.96 \times 10^{-12}$ & & $4.80 \pm 2.46$ & $-0.43 \pm 0.73$ \\
\hline Debiased & & & & $1998.8-2008.6$ & 396 & 2001.2 & $8.42 \pm 5.90 \times 10^{-12}$ & -0.63 & $3.33 \pm 2.38$ & $0.00 \pm 0.71$ \\
\hline Best $^{g}$ & & & & & & & $6.86 \pm 0.50 \times 10^{-12}$ & & $2.72 \pm 0.43$ & $0.19 \pm 0.13$ \\
\hline Raw & 9 Metis & $87 \pm 6^{h, j}$ & 29818 & 1996.5-2008.9 & 468 & 2005.2 & $13.1 \pm 6.33 \times 10^{-12}$ & & $9.47 \pm 4.98$ & $-1.83 \pm 1.49$ \\
\hline Debiased & & & & $1996.5-2008.9$ & 468 & 2005.2 & $4.54 \pm 0.86 \times 10^{-12}$ & -10.0 & $3.28 \pm 0.92$ & $0.02 \pm 0.27$ \\
\hline Best $^{\mathrm{g}}$ & & & & & & & $5.70 \pm 1.40 \times 10^{-12}$ & & $4.12 \pm 1.33$ & $-0.23 \pm 0.40$ \\
\hline Raw & 10 Hygiea & $215.3 \pm 3.4^{\mathrm{f}, \mathrm{h}}$ & 3946 & 1996.8-2008.9 & 1093 & 1998.2 & $7.15 \pm 5.80 \times 10^{-11}$ & & $3.40 \pm 2.77$ & $-0.15 \pm 0.94$ \\
\hline Debiased & & & & $1996.8-2008.9$ & 1093 & 1998.2 & $6.51 \pm 5.80 \times 10^{-11}$ & -0.11 & $3.10 \pm 2.77$ & $-0.05 \pm 0.94$ \\
\hline Best $^{\mathrm{g}}$ & & & & & & & $4.45 \pm 0.07 \times 10^{-11}$ & & $2.12 \pm 0.11$ & $0.28 \pm 0.04$ \\
\hline Raw & 10 Hygiea & $215.3 \pm 3.4^{\mathrm{f}, \mathrm{h}}$ & 24433 & 1995.1-2008.9 & 504 & 2004.5 & $4.11 \pm 0.85 \times 10^{-11}$ & & $1.96 \pm 0.42$ & $0.34 \pm 0.14$ \\
\hline Debiased & & & & $1995.1-2008.9$ & 504 & 2004.5 & $4.08 \pm 0.83 \times 10^{-11}$ & -0.04 & $1.94 \pm 0.41$ & $0.34 \pm 0.14$ \\
\hline Best $^{\mathrm{g}}$ & & & & & & & $4.45 \pm 0.07 \times 10^{-11}$ & & $2.12 \pm 0.11$ & $0.28 \pm 0.04$ \\
\hline Raw & 10 Hygiea & $215.3 \pm 3.4^{\mathrm{f}, \mathrm{h}}$ & 57493 & 1996.4-2007.5 & 314 & 2000.6 & $2.41 \pm 5.90 \times 10^{-11}$ & & $1.15 \pm 2.81$ & $0.61 \pm 0.95$ \\
\hline Debiased & & & & 1996.4-2007.5 & 314 & 2000.6 & $3.80 \pm 7.13 \times 10^{-11}$ & 0.19 & $1.81 \pm 3.40$ & $0.39 \pm 1.15$ \\
\hline Best $^{g}$ & & & & & & & $4.45 \pm 0.07 \times 10^{-11}$ & & $2.12 \pm 0.11$ & $0.28 \pm 0.04$ \\
\hline Raw & 10 Hygiea & $215.3 \pm 3.4^{\mathrm{f}, \mathrm{h}}$ & 75794 & $2000.0-2008.2$ & 208 & 2005.7 & $5.87 \pm 4.28 \times 10^{-11}$ & & $2.80 \pm 2.04$ & $0.05 \pm 0.69$ \\
\hline Debiased & & & & $2000.0-2008.2$ & 208 & 2005.7 & $4.06 \pm 3.96 \times 10^{-11}$ & -0.46 & $1.94 \pm 1.89$ & $0.34 \pm 0.64$ \\
\hline Best $^{\mathrm{g}}$ & & & & & & & $4.45 \pm 0.07 \times 10^{-11}$ & & $2.12 \pm 0.11$ & $0.28 \pm 0.04$ \\
\hline Raw & 15 Eunomia & $134.1 \pm 7.5^{\mathrm{f}, \mathrm{h}}$ & 50278 & 1998.9-2008.2 & 220 & 2002.2 & $1.65 \pm 0.13 \times 10^{-11}$ & & $3.24 \pm 0.60$ & $0.03 \pm 0.18$ \\
\hline Debiased & & & & 1998.9-2008.2 & 220 & 2002.2 & $1.59 \pm 0.13 \times 10^{-11}$ & -0.41 & $3.14 \pm 0.59$ & $0.06 \pm 0.17$ \\
\hline Best $^{g}$ & & & & & & & $1.57 \pm 0.02 \times 10^{-11}$ & & $3.09 \pm 0.52$ & $0.08 \pm 0.16$ \\
\hline Raw & 189 Phthia & $19 \pm 2^{\mathrm{h}}$ & 6224 & 1995.0-2008.9 & 547 & 1999.1 & $7.93 \pm 2.04 \times 10^{-14}$ & & $5.49 \pm 2.24$ & $-0.64 \pm 0.67$ \\
\hline Debiased & & & & 1995.0-2008.9 & 547 & 1999.1 & $7.06 \pm 2.20 \times 10^{-14}$ & -0.40 & $4.89 \pm 2.17$ & $-0.46 \pm 0.65$ \\
\hline Best $^{\mathrm{g}}$ & & & & & & & $1.93 \pm 0.41 \times 10^{-14}$ & & $1.34 \pm 0.51$ & $0.60 \pm 0.15$ \\
\hline
\end{tabular}

a The relative difference is the difference between the raw and debiased mass estimates, normalized by the stated debiased (formal) uncertainty.

${ }^{b}$ We assume grain densities of $2.4 \mathrm{~g} / \mathrm{cm}^{\mathrm{e}}$ for $\mathrm{S}$ types (i.e., asteroids 3, 6, 7, 9, 15 and 189 ) and $\mathrm{G}$ types (i.e., $1 \mathrm{Ceres),} \mathrm{and} 3.0 \mathrm{~g} / \mathrm{cm}^{\mathrm{e}}$ for $\mathrm{C}$ types (i.e., $10 \mathrm{Hygiea).}$

c References:Pitjeva (2005).

d Thomas et al. (2005).

e Millis et al. (1981).

${ }^{f}$ Kaasalainen et al. (2002).

g Baer et al. (2008).

h Tedesco et al. (2002).

i Torppa et al. (2003).

j Thomas et al. (1996).

laboratory and NeoDys ${ }^{6}$ at the University of Pisa (Milani et al., 2005), which continually monitor the ever-changing asteroid orbit catalog for impact possibilities and assess the associated probabilities. But to date these assessments have been obtained with raw, and generally unweighted, astrometry, and so these assessments will need to be re-run with catalog bias corrections applied. While we do not expect the new assessments to wholly invalidate the majority of current potential impacts, it will undoubtedly lead to many impacts possibilities being eliminated as no longer consistent with the new (debiased) astrometry, and to many new potential impacts that were previously not compatible with the original (raw) astrometry. For many cases that persist, there will be significant corrections to the tabulated probability of impact.

\subsubsection{Apophis}

Of particular interest is the Asteroid 99942 Apophis, discovered by R. Tucker, D. Tholen and F. Bernardi at Kitt Peak in June 2004, and subsequently lost due to weather and low solar elongation. It was serendipitously recovered by G. Garradd at Siding Spring in December 2004 (Gilmore et al., 2004). In the days following the rediscovery, it became clear that there would be a very close Earth approach in April 2029, and at least initially, the possibility

\footnotetext{
${ }^{6}$ http://newton.dm.unipi.it/neodys/.
}

of impact could not be ruled out. The impact probability reached a peak of $2.7 \%$ (Chesley, 2006) at which point pre-discovery measurements from March 2004 were reported (Gleason et al., 2004), which allowed the 2029 impact to be ruled out. Still, the 2029 close approach would be very close indeed, nominally at about 38,000 km from the geocenter on April 13, 2029.

At this point the key question became what would happen after 2029 , when the orbit would be deflected significantly, from the Aten to the Apollo class, and the orbital uncertainty would expand dramatically due to the scattering effect of the Earth close approach. Resonant return impacts (Milani et al., 1999) were possible in the years following 2029, but many of these were ruled out as the $2029 b$-plane uncertainty region was reduced through continuing observations. As of this writing, only the 2036 and 2037 potential impacts remain (7:6 and 8:7 resonances, respectively). These impacts will take place if the true asteroid $b$-plane intersection falls on a narrow region, called a keyhole, which can be defined as the intersection of the uncertainty region and the Valsecchi circle associated with the given resonance (Valsecchi et al., 2003). The key question here is how much the current $b$ plane footprint is affected by the introduction of catalog debiasing to the orbit determination.

The current Apophis orbit determination, JPL solution 140, is obtained with raw astrometry and uses manually adjusted data 


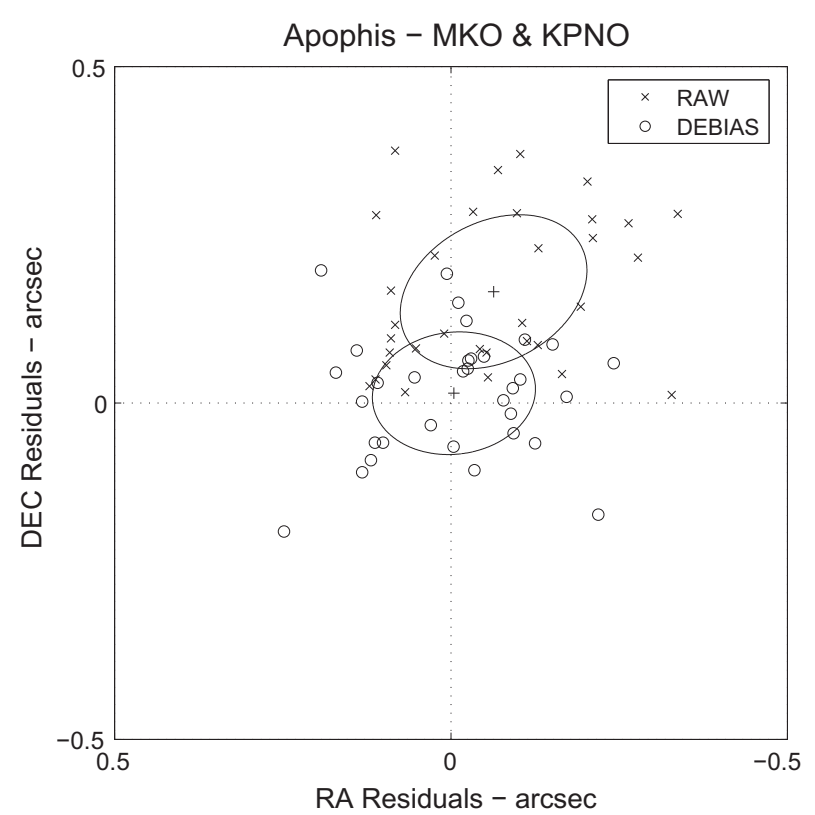

Fig. 18. For 99942 Apophis orbital fits, effect of debiasing on postfit residuals for Mauna Kea (568) and Kitt Peak (695) astrometry. One sigma covariance ellipses for the respective data sets are shown.

weighting reflective of rough estimates of the observation quality. The data set at present uses 738 optical measurements from 200403-15 to 2006-08-16, plus two radar delay and Doppler measurements (Giorgini et al., 2008). We have also performed a fit with debiased astrometry, using the same data set and weights as solution 140 . The postfit residuals for the raw solution show a significant positive offset in the declination residuals, especially for the later observations derived by Tholen et al. (2008). This trend apparently continues, as Tholen et al. (2008) report that they have reduced close to 200 additional, and so far unreported, observations through January 2008, which show a persistent positive declination bias. It is clear from inspection of the data that the astrometric reductions by Tholen et al. (2008), consisting of the observations made at Mauna Kea Observatory (MKO, code 568) as well as the discovery series of measurements from Kitt Peak National Observatory (KPNO, code 695), have an internal consistency far superior to other contributors of optical astrometry.

Through the MPC, we currently have access to a combined total of only 33 KPNO and MKO observations. The residuals for these observations, for both the raw and debiased fits, are plotted in Fig. 18. The plot shows the clear bias in the astrometry from the raw fit, where there is not even a single negative declination residual, and the mean is located at -0.5 and +1.5 standard deviations in RA and DEC, respectively. In contrast, the residuals from the debiased fit show effectively zero mean and reduced scatter as indicated by the smaller covariance ellipse. It is important to recognize that we have only plotted residuals for 33 of the 738 observations used in the fit, but the debiased fit has applied catalog bias corrections, according to the technique described earlier, to all observations. Thus the effect of debiasing seen in Fig. 18 comes from two directions: the orbit is improved so that orbital error no longer contributes to the declination bias problem, and the MKO and KPNO observations themselves are improved through debiasing. Comparable plots for other data sets are not as illuminating because none have such small internal noise levels, and so the associated ellipses are much larger than those seen in Fig. 18. Even so, the clear conclusion, which is to be expected by now, is that the debiased astrometry allows for more consistent fits, which should lead to better predictions.
Fig. 19 reveals the effect of debiasing on the $2029 b$-plane intersection, and specifically the keyhole overlap. We use the $b$-plane formalism and notation described by Valsecchi et al. (2003), where the Earth's inertial velocity projects onto the $-\zeta$-axis. The raw and debiased Apophis prediction ellipses, with one-sigma semiaxes of $15 \times 560 \mathrm{~km}$, are noticeably offset from each other. The offsets are $(\Delta \xi, \Delta \zeta) \simeq(23,-170) \mathrm{km}$, which corresponds to $1.7 \sigma$ in $\xi$ and $0.3 \sigma$ in $\zeta$. While the $\xi$ offset is small in absolute terms, it is by far the larger in a relative sense, and this is because the catalog bias is dominated by declination errors, and so leads to errors in inclination, which map primarily into the $\xi$ direction. From the figure, we can see that the effect of debiasing on the $2029 \mathrm{~b}$-plane prediction is to move the prediction region away from the 2036 Valsecchi circle, downgrading the threat from the associated potential impact, but slightly upgrading that from the 2037 impact. We refer to the long axis of the ellipse as the Line of Variations (LOV), and it is informative to place the keyholes in the frame of the LOV, measured in sigmas. The LOV positions, and associated impact probabilities, computed as described in Milani et al. (2002) are tabulated in Table 13.

While we do not at this point have access to the unpublished astrometry described by Tholen et al. (2008), we can coarsely estimate the effect of these data by simulating 16 nights of data, with three positions each night, totaling 48 positions. The 16 nights selected are the 15th, 16th, 17th and 18th of December 2008, January 2007, December 2007 and January 2008, and we weight the simulated data at 0.3 arcsec, just as we have weighted the other astrometry from MKO. These simulated observations reduce the major axis of the $b$-plane ellipse by $28 \%$, while reducing the minor axis by only $6 \%$. The impact threat derived with the additional simulated data is given under the label "Future" in Table 13. The results indicate that the unpublished astrometry has the power to dramatically reduce the impact risk from Apophis, assuming that it does not significantly alter the new orbital solution. This goes to the question of orbital stability, and fits over different time intervals indicate clearly that the debiased fits are very stable as new data are added, while the raw fits show a steady walk (towards the debiased solution) in the $2029 b$-plane as new data are added.

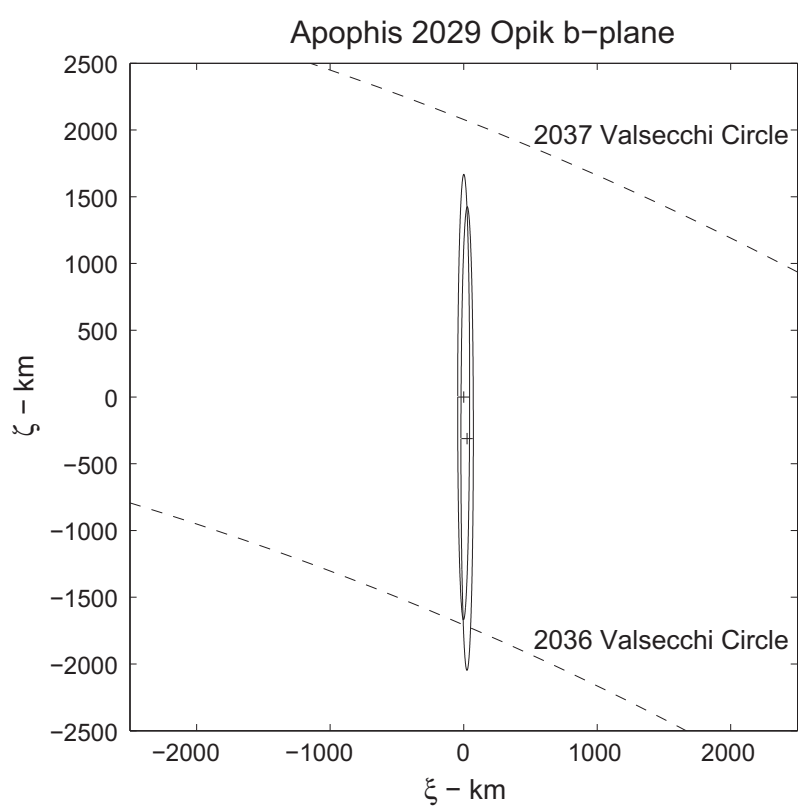

Fig. 19. Mapping of raw and debiased Apophis orbital solutions to the 2029 Öpik $b$ plane, with 3-sigma confidence ellipses. The debiased prediction (upper ellipse) is at the origin in this depiction and the Earth is at $(\xi, \zeta) \simeq(-9500,-48,000) \mathrm{km}$. The Valsecchi circles, which lead to a resonant return and subsequent impact are also depicted. 
Table 13

Apophis impacts in 2036 and 2037.

\begin{tabular}{|c|c|c|c|c|c|}
\hline \multirow[t]{2}{*}{ Case } & \multirow[t]{2}{*}{ b-Plane semimajor axis ( $\mathrm{km})$} & \multicolumn{2}{|l|}{ April 2036} & \multicolumn{2}{|l|}{ April 2037} \\
\hline & & LOV sigma & IP & LOV sigma & IP \\
\hline Raw (JPL\#140) & 560 & -2.44 & $2.2 \times 10^{-5}$ & 4.11 & $8.1 \times 10^{-8}$ \\
\hline Debiased & 560 & -3.02 & $4.5 \times 10^{-6}$ & 3.53 & $8.2 \times 10^{-7}$ \\
\hline Future $^{\mathrm{a}}$ & 400 & -4.2 & $8.8 \times 10^{-8}$ & 4.9 & $3.5 \times 10^{-9}$ \\
\hline \multicolumn{6}{|l|}{ With Yarkovsky ${ }^{\mathrm{b}}$} \\
\hline Debiased & 650 & -2.66 & $1.1 \times 10^{-5}$ & 3.10 & $2.9 \times 10^{-6}$ \\
\hline Future $^{\mathrm{a}}$ & 520 & -3.4 & $1.7 \times 10^{-6}$ & 3.9 & $2.1 \times 10^{-7}$ \\
\hline
\end{tabular}

a Assumes debiased solution is unaltered by addition of 48 observations described in text.

b Assumes Gaussian dispersions of $10 \times 10^{-4} \mathrm{AU} / \mathrm{Myr}$.

So far we have not considered nongravitational accelerations in the trajectory of Apophis, but we are reaching the point where this is starting to become necessary. Chesley (2006) first pointed to the future importance of the Yarkovsky effect (Bottke et al., 2006) in assessing the threat posed by Apophis. Later, Giorgini et al. (2008) conducted a thorough review of the prediction uncertainties associated with the Apophis dynamical model, showing that the Yarkovsky effect is indeed the dominant source of dynamical modeling error, with potentially hundreds of kilometers of offset in the $2029 b$-plane due to Yarkovsky accelerations, which is comparable to the current $b$-plane uncertainty. The problem at hand is that, lacking information on the obliquity of the Apophis spin axis, we do not even know whether the Yarkovsky effect is causing the semimajor axis to increase or decrease. Chesley (2006) found that the semimajor axis drift rate $d a / d t$ for Apophis should be in the range of $\pm 15 \times 10^{-4} \mathrm{AU} / \mathrm{Myr}$, but later information suggests that the object may be somewhat smaller than originally assumed (Delbò et al., 2007), thus increasing the effect. In order to have a rudimentary understanding of how the Yarkovsky effect might affect the impact risk we take a Gaussian distribution on $d a / d t$ with one-sigma at $10 \times 10^{-4} \mathrm{AU} / \mathrm{Myr}$ and obtain a revised $b$-plane footprint. With this dispersion due to Yarkovsky, the $b$-plane semimajor axis grows by a modest amount as indicated in Table 13, which moves the keyholes closer to nominal, in a relative sense, thereby increasing the impact probability.

The Yarkovsky dispersions assumed here are unacceptably simplistic, and the computed impact probabilities suffer accordingly. But the exercise serves to emphasize the fact that Yarkovsky perturbations have become the key source of uncertainty in assessing the hazard posed by Apophis. We intend to revisit this question in the near future with a more rigorous analysis that includes additional astrometry and an empirical distribution on $d a / d t$.

\section{Conclusions}

We have demonstrated that our debiasing algorithm significantly reduces the biases in the observational residuals. We have also seen that only closely-spaced observations of the same object made by the same observatory remain correlated. However, these remaining correlations are significant; and an accurate observational error model must account for them.

The approach demonstrated in Section 6.1, while an improvement over conventional techniques, is sub-optimal, since it essentially doubles our best estimate of the observational uncertainties, in order to compensate for the unmodeled correlations.

Additionally, we note that combining observations from existing surveys with the forthcoming highly-precise astrometric data from PanSTARRS in the same least-squares solutions will require the use of realistic observational uncertainties; simply assuming that all observatories have the same observational uncertainties will eliminate any improvements that might have resulted from the more precise PanSTARRS data.
Our intent, therefore, is to use the debiased residual statistics to develop observatory-specific, time-based models of observational uncertainties and correlations, as described by Carpino et al. (2003). The resulting best-fit orbits should allow us to further refine our estimates of asteroid masses, densities, and porosities, improve detection of Yarkovsky effects, and yield more reliable collision analyses.

The same principles could also be applied to develop a statistical model of asteroid photometry, potentially yielding more precise shape models and rotational periods. Combined with the observational error model, we should be able to glean the maximum information as to asteroid physical compositions, structures, and dynamical states from the observational database.

\section{Note added in proof}

This analysis of the Apophis impact probability is included primarily as an indication of the potential effect of debiased astrometry. The details of the impact assessment have been updated with new astrometry and a complete consideration of Yarkovsky accelerations by Chesley et al. (2009).

\section{Acknowledgments}

We are grateful to Tim Spahr and Gareth Williams of the Minor Planet Center at Harvard Univ., who provided the astrometric catalog information, which was vital for this effort to proceed. Numerous observers and observing programs provided valuable information beyond what is available from the MPC data files. In particular, we thank Bob McMillan (Spacewatch), Ed Beshore (Catalina Sky Survey), Scott Stuart (LINEAR), Bruce Koehn (LONEOS) and Ken Lawrence (NEAT) for their cooperation. Finally, we are sincerely grateful to Andrea Milani, for his expertise and help at each step of this effort. This publication makes use of data products from the Two Micron All Sky Survey, which is a joint project of the University of Massachusetts and the Infrared Processing and Analysis Center/California Institute of Technology, funded by the National Aeronautics and Space Administration and the National Science Foundation. Some of the results in this paper have been derived using the HEALPix package (Górski et al., 2005). This research was conducted in part at the Jet Propulsion Laboratory, California Institute of Technology, under a contract with the National Aeronautics and Space Administration.

\section{References}

Arias, E.F., Charlot, P., Feissel, M., Lestrade, J., 1995. The extragalactic reference system of the International Earth Rotation Service, ICRS. Astron. Astrophys. 303, 604-608.

Baer, J., Milani, A., Chesley, S., Matson, R.D., 2008. An observational error model, and application to asteroid mass determination. Bull. Am. Astron. Soc. 40, 493.

Bottke Jr., W.F., Vokrouhlický, D., Rubincam, D.P., Nesvorný, D., 2006. The Yarkovsky and Yorp effects: Implications for asteroid dynamics. Annu. Rev. Earth Planet. Sci. 34, 157-191. 
Carpino, M., Milani, A., Chesley, S.R., 2003. Error statistics of asteroid optical astrometric observations. Icarus $166,248-270$.

Chesley, S.R., 2006. Potential impact detection for near-Earth asteroids: The case of 99942 Apophis (2004 MN 4). In: Daniela, L., Sylvio Ferraz, M., Angel, F.J. (Eds.), Asteroids, Comets, Meteors, IAU Symposium, vol. 229, pp. 215-228.

Chesley, S. et al., 2009. An Updated Assessment of the Impact Threat from 99942 Apophis. Div. Planetary Sciences Mtg., October.

da Silva Neto, D.N., Andrei, A.H., Assafin, M., Vieira Martins, R., 2005. Investigation on the southern part of the high density astrometric catalogs USNO B1.0, 2MASS and UCAC2. Astron. Astrophys. 429, 739-745.

Delbò, M., Cellino, A., Tedesco, E.F., 2007. Albedo and size determination of potentially hazardous asteroids: (99942) Apophis. Icarus 188, 266-269.

Gilmore, A.C., and 11 colleagues, 2004. 2004 MN4. Minor Planet Electronic Circulars, p. 25.

Giorgini, J.D., Benner, L.A.M., Ostro, S.J., Nolan, M.C., Busch, M.W., 2008. Predicting the Earth encounters of (99942) Apophis. Icarus 193, 1-19.

Gleason, A.E., Larsen, J.A., Descour, A.S., Williams, G.V., 2004. 2004 MN4. Minor Planet Electronic Circulars, p. 70.

Górski, K.M., Hivon, E., Banday, A.J., Wandelt, B.D., Hansen, F.K., Reinecke, M. Bartelmann, M., 2005. HEALPix: A framework for high-resolution discretization and fast analysis of data distributed on the sphere. Astrophys. J. 622, 759-771.

Høg, E., Fabricius, C., Makarov, V.V., Urban, S., Corbin, T., Wycoff, G., Bastian, U. Schwekendiek, P., Wicenec, A., 2000. The Tycho-2 catalogue of the 2.5 million brightest stars. Astron. Astrophys. 355, L27-L30.

Jedicke, R., and the Pan-Starrs Collaboration, 2004. The Pan-STARRS Solar System survey. In: 35th COSPAR Scientific Assembly, COSPAR, Plenary Meeting, vol. 35, p. 999.

Kaasalainen, M., Torppa, J., Piironen, J., 2002. Models of twenty asteroids from photometric data. Icarus 159, 369-395.

Milani, A., Chesley, S.R., Chodas, P.W., Valsecchi, G.B., 2002. Asteroid close approaches: Analysis and potential impact detection. Asteroids III, 55-69.

Milani, A., Chesley, S.R., Sansaturio, M.E., Tommei, G., Valsecchi, G.B., 2005 Nonlinear impact monitoring: Line of variation searches for impactors. Icarus $173,362-384$.

Milani, A., Chesley, S.R., Valsecchi, G.B., 1999. Close approaches of Asteroid $1999 \mathrm{AN}_{10}$ : Resonant and non-resonant returns. Astron. Astrophys. 346, L65-L68.
Millis, R.L., and 10 colleagues, 1981. The diameter of Juno from its occultation of AG +0 deg 1022. Astron. J. 86, 306-313.

Monet, D., 1998. README File for the USNO-A2 Catalogs. <http://www.usno. navy.mil/USNO/astrometry/optical-IR-prod/icas/the-pmm/readme.v20>.

Monet, D., Canzian, B., Harris, H., Reid, N., Rhodes, A., Sell, S., 1998. The PMM USNOA1.0 Catalogue (Monet 1997). VizieR Online Data Catalog, p. 1243.

Monet, D.G., and 28 colleagues, 2003. The USNO-B catalog. Astron. J. 125, $984-$ 993.

Pitjeva, E.V., 2005. High-precision ephemerides of planets - EPM and determination of some astronomical constants. Solar Syst. Res. 39, 176-186.

Skrutskie, M.F., and 30 colleagues, 2006. The two micron all sky survey (2MASS). Astron. J. 131, 1163-1183.

Stokes, G.H., Evans, J.B., Viggh, H.E.M., Shelly, F.C., Pearce, E.C., 2000. Lincoln nearEarth asteroid program (LINEAR). Icarus 148, 21-28.

Tedesco, E.F., Noah, P.V., Noah, M., Price, S.D., 2002. The supplemental IRAS minor planet survey. Astron. J. 123, 1056-1085.

Tholen, D.J., Bernardi, F., Micheli, M., 2008. Where is Apophis? Bull. Am. Astron. Soc. $40,434$.

Thomas, P.C., Belton, M.J.S., Carcich, B., Chapman, C.R., Davies, M.E., Sullivan, R. Veverka, J., 1996. The shape of Ida. Icarus 120, 20-32.

Thomas, P.C., Parker, J.W., McFadden, L.A., Russell, C.T., Stern, S.A., Sykes, M.V., Young, E.F., 2005. Differentiation of the Asteroid Ceres as revealed by its shape. Nature 437, 224-226.

Torppa, J., Kaasalainen, M., Michalowski, T., Kwiatkowski, T., Kryszczyńska, A., Denchev, P., Kowalski, R., 2003. Shapes and rotational properties of thirty asteroids from photometric data. Icarus 164, 346-383.

Williams, G., 2009. IAU Minor Planet Center. <http://www.cfa.harvard.edu/iau/ mpc.html>.

Valsecchi, G.B., Milani, A., Gronchi, G.F., Chesley, S.R., 2003. Resonant returns to close approaches: Analytical theory. Astron. Astrophys. 408, 11791196.

Zacharias, N., Urban, S.E., Zacharias, M.I., Wycoff, G.L., Hall, D.M., Monet, D.G., Rafferty, T.J., 2004. The second US Naval Observatory CCD astrograph catalog (UCAC2). Astron. J. 127, 3043-3059.

Zacharias, N., and 29 colleagues, 2004. The Third US Naval Observatory CCD Astrograph Catalog (UCAC3). ArXiv e-prints, 1003.2136. 